\title{
Prophylactic ulinastatin administration for preventing post-endoscopic retrograde cholangiopancreatography pancreatitis: A meta-analysis
}

\author{
KUN ZHU, JIAN-PING WANG and JIN-GEN SU \\ Department of Surgery, Shanghai Jiading Central Hospital, Shanghai 201800, P.R. China
}

Received August 2, 2016; Accepted May 5, 2017

DOI: $10.3892 /$ etm.2017.4910

\begin{abstract}
The objective of the present study was to perform a meta-analysis of all available studies on the effect of prophylactic ulinastatin administration on preventing post-endoscopic retrograde cholangiopancreatography (ERCP) pancreatitis (PEP). The PubMed, Web of Knowledge and Chinese National Knowledge Infrastructure databases were searched to identify all relevant studies published in English or Chinese prior to April 2016. Cochrane Review Manager was used to calculate the pooled risk ratio (RR) and $95 \%$ confidence interval (CI) to determine the effect of prophylactic ulinastatin on PEP, post-ERCP hyperamylasemia (PEHA) and post-ERCP abdominal pain. The analysis revealed that prophylactic ulinastatin administration significantly reduced the PEP risk $(\mathrm{RR}=0.49$; 95\% CI: 0.33-0.74; $\left.\mathrm{P}=0.0006 ; \mathrm{I}^{2}=24\right)$; however, such significant risk reduction occurred only in patients with low or average risk for PEP and high-dosage ulinastatin (150,000 or 200,000 U) administration, and when the ulinastatin administration began prior to or during ERCP. Pre-ERCP ulinastatin administration alone without additional administration after ERCP was sufficient. Prophylactic ulinastatin also significantly reduced the PEHA risk $(\mathrm{RR}=0.68 ; 95 \% \mathrm{CI}$ : 0.56-0.83; $\mathrm{P}=0.0001$; $\left.\mathrm{I}^{2}=19\right)$ and marginally reduced the incidence of post-ERCP abdominal pain $\left(\mathrm{RR}=0.67\right.$; 95\% CI: 0.45-1.00; $\left.\mathrm{P}=0.05 ; \mathrm{I}^{2}=67\right)$. In conclusion, prophylactic ulinastatin administration significantly reduced the risk of PEP in patients with low or average risk for PEP when administered at a high dosage prior to or during ERCP. High-quality studies, particularly on high-risk patients, are warranted.
\end{abstract}

Correspondence to: Dr Kun Zhu, Department of Surgery, Shanghai Jiading Central Hospital, 1 Chengbei Road, Jiading, Shanghai 201800, P.R. China

E-mail: kunzhushanghai@sina.com

Key words: post-endoscopic retrograde cholangiopancreatography pancreatitis, ulinastatin, hyperamylasemia, meta-analysis, abdominal pain

\section{Introduction}

Endoscopic retrograde cholangiopancreatography (ERCP) has been a widely accepted procedure for diagnosing and evaluating pancreatic and biliary diseases ever since its introduction in the 1970s, and it remains the first choice in treating and managing various pancreaticobiliary diseases $(1,2)$. However, it also has the disadvantage of carrying the highest complication rate among all of the gastrointestinal endoscopic procedures currently in practice $(1,2)$. Post-ERCP complications include pancreatitis, cholangitis, cholecystitis, perforation and bleeding; among them, post-ERCP pancreatitis (PEP) is most common. Its estimated incidence ranges from $1-10 \%$ in average-risk patients, and reaches nearly $30 \%$ in high-risk patients $(1,2)$. Although PEP is mild or moderate in most of the cases, it is severe in $\sim 10 \%$ of the cases and may potentially be fatal $(3,4)$. Given the relatively high incidence of PEP and its potentially severe consequences, it is no surprise that the interest in developing pharmaceutical preventions for PEP has been growing (2). However, studies on the preventive effects of various pharmaceutical agents on PEP often produced inconsistent or even conflicting results and therefore, the 2010 European Society of Gastrointestinal Endoscopy (ESGE) guidelines for the prophylaxis of PEP stated that non-steroidal anti-inflammatory drugs are the only ones with proven efficacy (5).

Since proteolytic enzyme activation starting with activation of trypsinogen to trypsin in pancreatic acinar cells has a triggering and important role in the pathogenesis of PEP, protease inhibitors such as ulinastatin and gabexate mesylate are potential prophylactic agents for reducing the risk of PEP, as ulinastatin, a trypsin inhibitor, may prevent the initial phase of the pathogenesis of PEP $(1,3,5)$. In fact, protease inhibitors such as ulinastatin and gabexate mesylate have already been approved by the Ministry of Health, Labor, and Welfare of Japan as therapeutic medications for acute pancreatitis, and they have been widely used in China and Japan to treat and manage acute pancreatitis $(1,5)$. Compared to gabexate mesylate that has a 55-second plasma half-life, ulinastatin has a relatively long plasma half-life (35 $\mathrm{min})$ and may be injected as a bolus as opposed to the 13-h continuous infusion required for gabexate mesylate to prevent pancreatic injury $(1,3,6)$. Despite being commonly used in Japan for PEP prevention, numerous studies on the efficacy of prophylactic ulinastatin 
in preventing PEP have produced inconsistent results (4,6-17). Several meta-analyses on this topic also produced inconsistent results, with the study by Chen et al (3) published in 2010 finding ulinastatin effective in reducing the incidence of PEP and post-ERCP hyperamylasemia (PEHA), an early indication of possible PEP, while the study by Yuhara et al (1) published in 2014 concluded that ulinastatin was not associated with a reduced risk of PEP. Since then, several additional studies on the effect of ulinastatin in preventing PEP and PEHA have been published. Probably due to their inconsistent results, protease inhibitors such as ulinastatin were not recommended in the 2010 ESGE guidelines.

The present study performed a meta-analysis of all available relevant studies on the effect of prophylactic ulinastatin on preventing PEP, PEHA and also on post-ERCP abdominal pain, another early indication of possible PEP. The present analysis may provide further evidence as to whether ulinastatin may be used as a prophylactic medication for PEP.

\section{Materials and methods}

Literature search. The PubMed, Web of Knowledge and Chinese National Knowledge Infrastructure (CNKI) databases were systematically searched to identify all relevant studies published prior to April 2016 in English or Chinese. The following search terms were used: 'post-endoscopic retrograde cholangiopancreatography', 'ERCP', 'pancreatitis', 'PEP', 'ulinastatin', 'urinary trypsin inhibitor' and 'UTI'. In addition, the reference lists of the identified studies and relevant reviews were examined to identify eligible studies possibly missed in the initial search.

Inclusion and exclusion criteria. All studies comparing the effect of prophylactic ulinastatin administration with a control in preventing PEP published in English or Chinese were considered eligible for the present meta-analysis. The inclusion criteria were as follows: i) The study was a controlled trial wherein patients were grouped into 2 treatment arms to receive either ulinastatin or a non-ulinastatin control (placebo or nothing), with all other treatments and medications being the same or comparable; ii) patients in the study received ERCP; and iii) the study reported on at least one of the following outcome measures: Incidence of PEP, PEHA or post-ERCP abdominal pain in the 2 groups.

Abstracts, reviews, editorials and studies without a proper control or with only one treatment arm were excluded.

KZ and JPW did a first round of screening by independently reviewing titles and abstracts of the studies identified by the initial search in order to shorten the list of relevant studies. They then reviewed the full-text studies on the shortened list to obtain a final list of studies to be included in the meta-analysis. Any disagreement between the two investigators was resolved by discussions among all of the contributing authors in order to reach a consensus.

Assessment of study quality. KZ and JPW independently assessed the quality of each included study according to Jadad et al (18) based on the following points: i) Whether the trial is randomized and if it is, whether the randomization is appropriate; ii) whether the study is double-blinded and if it is, whether the double-blinding is done appropriately; iii) whether there is a proper description of patient withdrawal or dropout, using a score of 0 or 1 for each question. Therefore, a study's possible total Jadad score were within the range of $0-5$. In the present meta-analysis, studies with a Jadad score of $\geq 3$ were defined as a high-quality studies, those with a Jadad score of 2 as average-quality studies and studies with a Jadad score $<2$ as low-quality studies.

Data extraction. $\mathrm{KZ}$ and JGS independently reviewed the full text of each study and extracted the following data: First author name, year of publication, study design, sample size (ulinastatin/control), male/female ratio, route and time of drug administration and main outcome measures. In addition, for the meta-analysis on the effect of ulinastatin on PEP, the definition of PEP, incidence of PEP and summary results were also extracted; for the meta-analysis on the effect on PEHA, definition of PEHA, incidence of PEHA and summary results were extracted; and for the meta-analysis on the effect of ulinastatin on post-ERCP abdominal pain, the incidence of post-ERCP abdominal pain and summary results were also extracted. The Jadad score of each included study was also determined. Any disagreement between the two reviewers was resolved by discussions between them.

Statistical analysis. Cochrane Review Manager (Revman, version 5.3; the Cochrane Institute, London, UK) was used to perform the statistical analysis. Pooled risk ratio (RR) and $95 \%$ confidence interval (CI) were used as the effect size measurements for the analysis, and they were first calculated with a random-effects model, as such a model expects and assumes a true diversity in the results of the included studies and hence includes a between-studies variance into its calculation (19). the $\mathrm{Z}$ test was used to assess the statistical significance of the pooled RRs, and a 95\% CI not including the value of 1 and $\mathrm{P}<0.05$ were considered to indicate statistical significance. Inter-study heterogeneities were assessed using the $\chi^{2}$ test based on Cochrane $\mathrm{Q}$ statistics and when a $\mathrm{P}$ value for the $\mathrm{Q}$ statistics was $<0.10$, the heterogeneity was statistically significant (20). The degree of inter-study heterogeneities was further quantified using the $\mathrm{I}^{2}$ index, with a higher $\mathrm{I}^{2}$ value representing a greater degree of heterogeneity (an $\mathrm{I}^{2}$ value of $\sim 25, \sim 50$ and $\sim 75 \%$ indicated low, moderate and high degree of heterogeneity, respectively) (21). If no inter-study heterogeneity was found using the random-effects model, pooled RR and 95\% CI were re-calculated using a fixed-effects model.

Sub-group analyses were performed for each of the 3 outcome measures based on the following factors: i) Whether the ulinastatin administration began prior to, during or after ERCP; ii) for those studies in which ulinastatin administration began prior to ERCP, whether ulinastatin was administered only prior to or also after ERCP; iii) whether the patients were at high risk of PEP; and iv) whether low- or high-dosage ulinastatin was administered. In addition, as part of a sensitivity analysis, sub-group analysis was also performed based on the qualities of the included studies in order to determine the impact of low-quality studies on the results and the stability and robustness of the analysis results. Sensitivity analysis further included change of the statistical model (a fixed-effects vs. a random-effects model). Finally, publication bias in the analysis was determined using a funnel plot. 

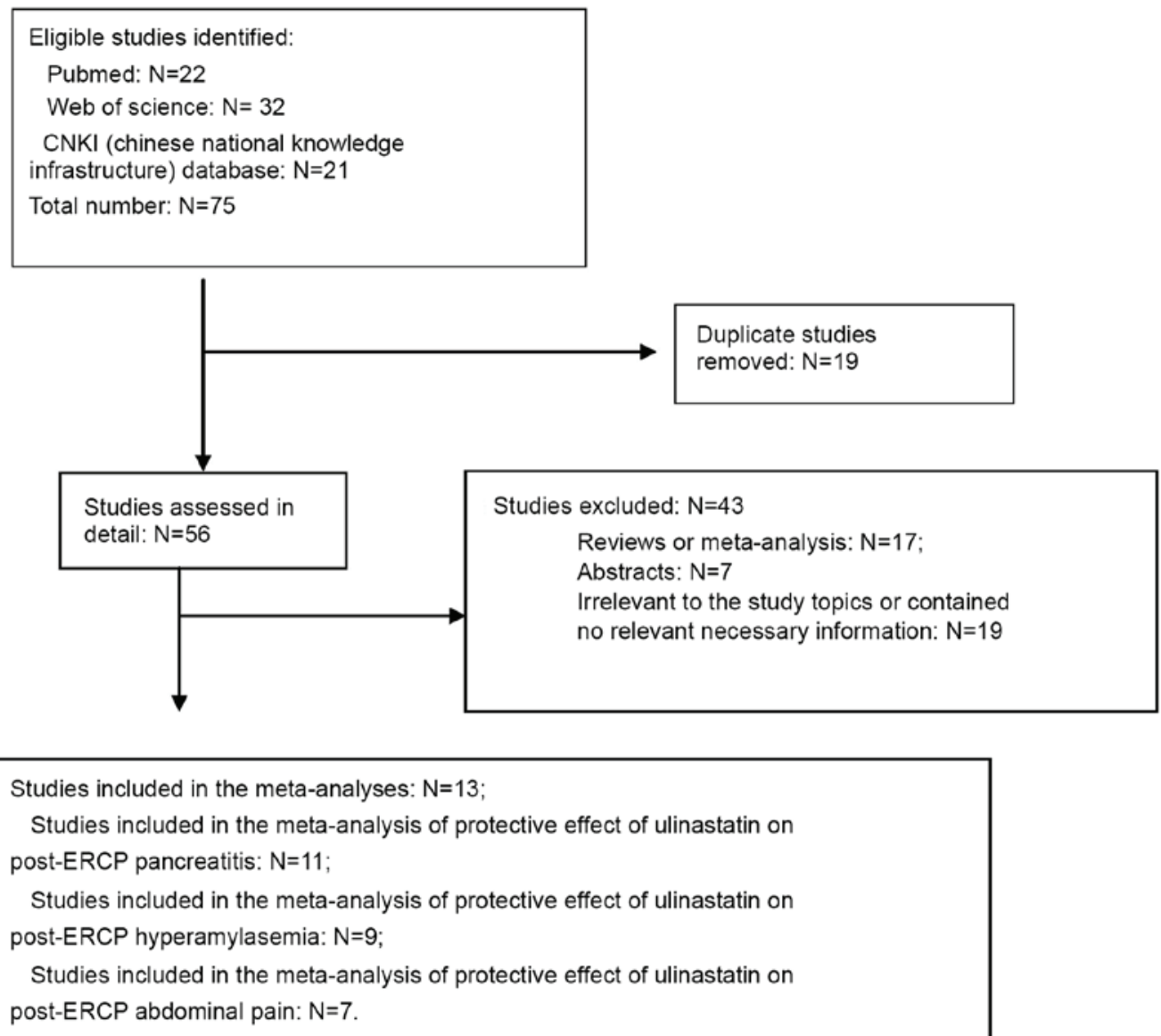

Figure 1. Flow diagram of the selection process of studies included in the present meta-analysis.

\section{Results}

Eligible studies and study characteristics. Fig. 1 presents a flow chart illustrating the literature search process of the present study and its results. A total of 75 potentially eligible studies were identified by searching the PubMed, Web of Science and CNKI databases. After excluding 19 duplicate studies, 56 studies were further examined to determine whether they meet the inclusion criteria and should be included in the present meta-analyses. A total of 43 studies were excluded, as they were reviews or meta-analyses, abstracts, irrelevant to the topic of the present study or did not provide any of the required data. Finally, 13 studies were included the present meta-analyses, and their characteristics are described in Table I. Among these 13 studies, 11 studies $(4,6-9,11-14,16,17)$ were included in the present meta-analysis on the effect of prophylactic ulinastatin on PEP, 9 studies $(4,6,8,9,11,13,14,16,17)$ were included in the meta-analysis on the effect of prophylactic ulinastatin on PEHA and 7 studies $(6,8-10,15-17)$ were included in the analysis on the effect of prophylactic ulinastatin on post-ERCP abdominal pain; their additional features were further described in Tables II-IV, respectively.

Effect of prophylactic ulinastatin administration on preventing PEP. As presented in Table II, 11 studies, including 1,103 patients who received prophylactic ulinastatin treatment and 1,463 patients who received placebo or no drug as a control were included in the meta-analysis on the efficacy of prophylactic ulinastatin in PEP prevention $(4,6-9,11-14,16,17)$. Prophylactic ulinastatin led to a significantly reduced risk of PEP compared with the control (RR=0.49; 95\% CI: 0.33-0.74; $\left.\mathrm{P}=0.0006 ; \mathrm{I}^{2}=24\right)$; however, such significant risk reduction only occurred when ulinastatin administration began prior to ( $\mathrm{RR}=0.46 ; 95 \% \mathrm{CI}$ : $0.26-0.83 ; \mathrm{P}=0.010 ; \mathrm{I}^{2}=32$ ) or during ERCP (RR=0.37; 95\% CI: 0.19-0.75; $\mathrm{P}=0.005 ; \mathrm{I}^{2}=0$ ), but not after ERCP (RR=0.64; 95\% CI: 0.15-2.70; $\mathrm{P}=0.55 ; \mathrm{I}^{2}=60$; Fig. 2A). In addition, ulinastatin administration prior to ERCP without additional administration after ERCP appeared to be sufficient to significantly reduce the PEP risk $(\mathrm{RR}=0.37$; 95\% CI: 0.15-0.90; $\mathrm{P}=0.03 ; \mathrm{I}^{2}=0$; Fig. $2 \mathrm{~B}$ ).

Further sub-group analysis revealed that the significantly reduced PEP risk associated with ulinastatin vs. control only occurred in patients with a low or average risk for PEP ( $\mathrm{RR}=0.49 ; 95 \%$ CI: $0.33-0.72 ; \mathrm{P}=0.0003 ; \mathrm{I}^{2}=13$ ), but not in high-risk patients $\left(\mathrm{RR}=0.29 ; 95 \%\right.$ CI: $0.01-6.37 ; \mathrm{P}=0.43 ; \mathrm{I}^{2}=87$; Fig. $2 \mathrm{C}$ ). The significant PEP risk reduction effect of ulinastatin was only observed with high-dosage ulinastatin administration $(150,000$ or $200,000 \mathrm{U}$; RR $=0.49 ; 95 \%$ CI: $0.32-0.74 ; \mathrm{P}=0.0008$; $\left.\mathrm{I}^{2}=19\right)$, but not with low-dosage ulinastatin $(100,000 \mathrm{U}$; $\mathrm{RR}=0.44 ; 95 \%$ CI: $0.10-1.88 ; \mathrm{P}=0.27 ; \mathrm{I}^{2}=55$; Fig. 2D).

As a part of the sensitivity analysis, the present study evaluated the impact of the qualities of the studies on the results by dividing the studies into high- or average-quality studies (Jadad score, $\geq 2$ ) and low-quality studies (Jadad score, $<2$ ). The sub-group analysis revealed that the qualities of the 


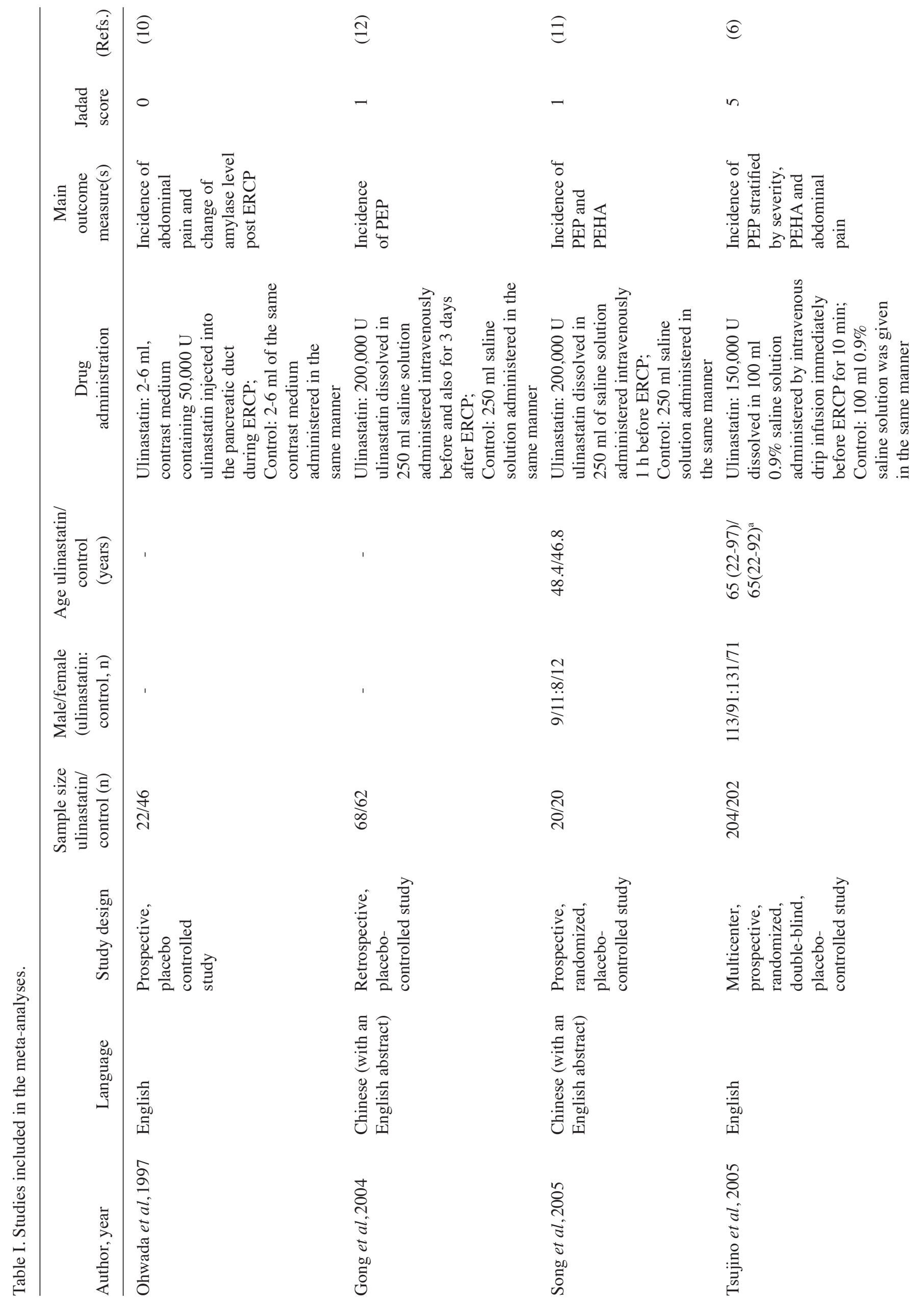




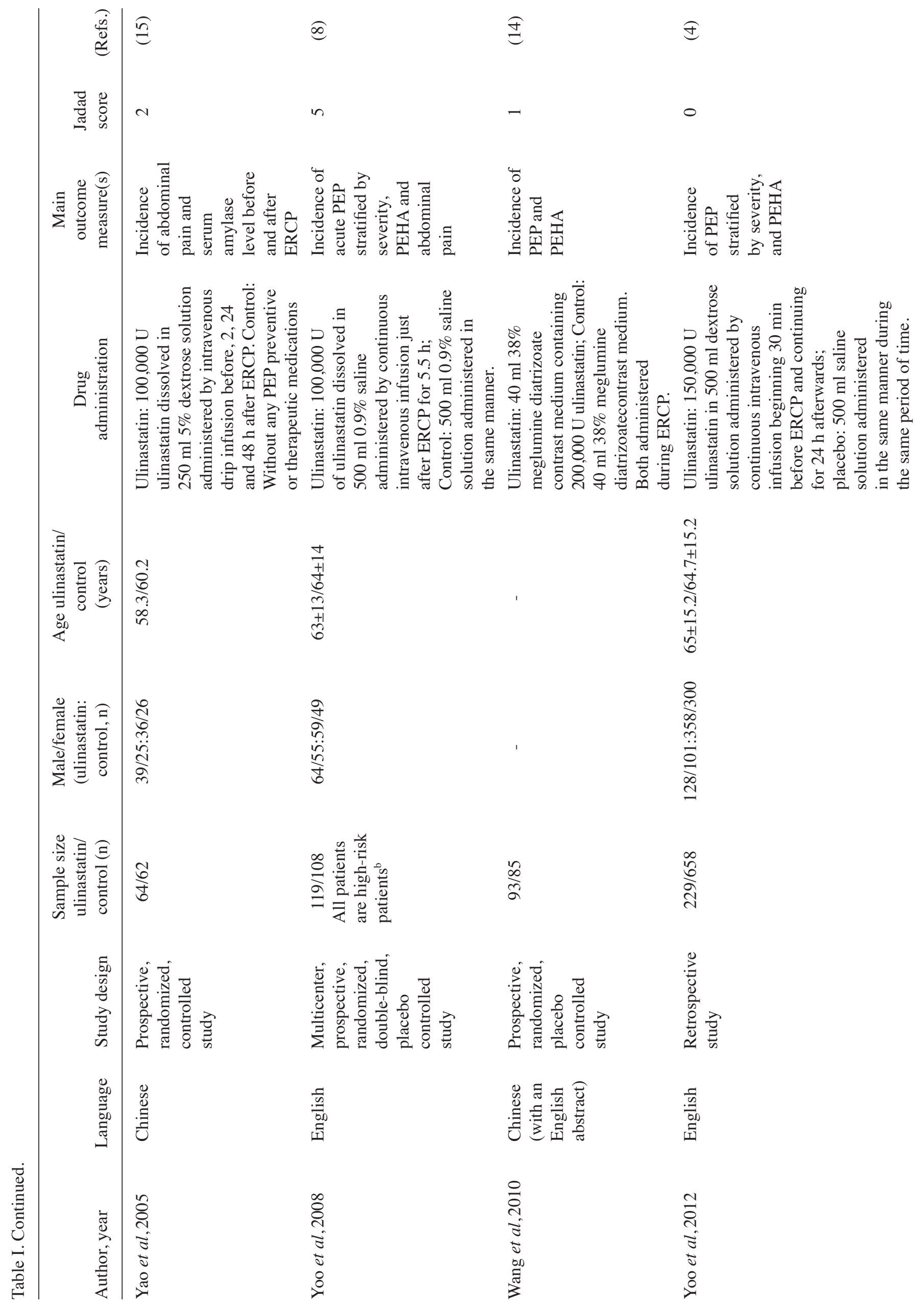




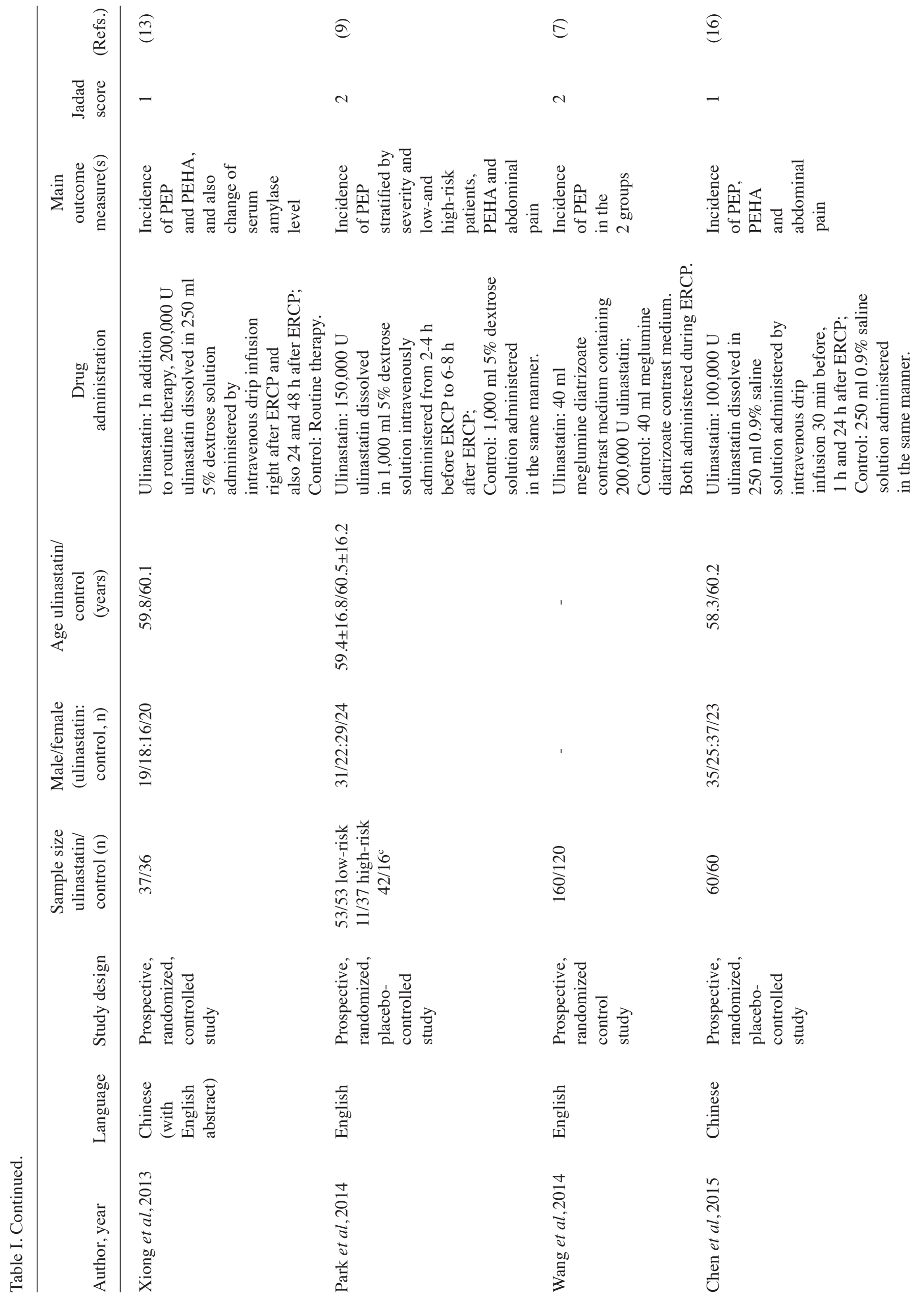




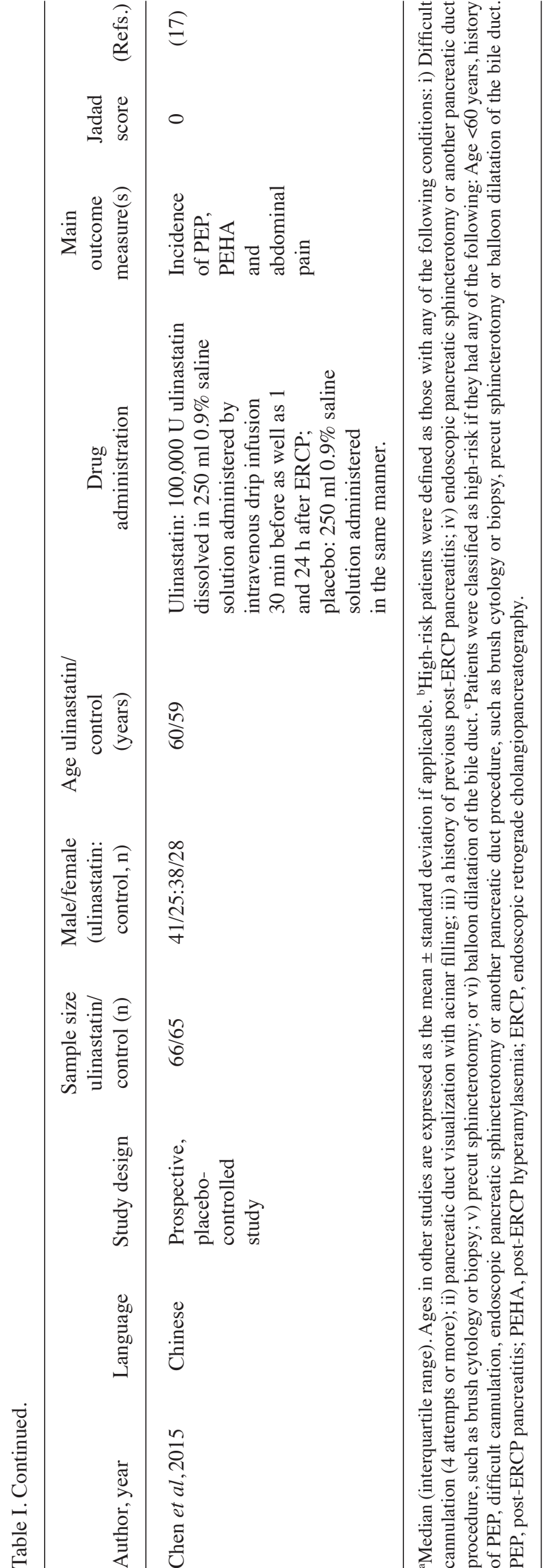

studies did not have any significant impact on the overall results, as analyses only using high- or average-quality studies and low-quality studies both showed a significant reduction of the PEP risk associated with ulinastatin vs. control (Fig. 2E).

The sensitivity analysis further included a change of the statistical model (fixed-effects vs. random-effects model). In most cases, using a fixed-effects model (not taking into account inter-study heterogeneities) did not significantly impact the results obtained using a random-effects model, indicating overall stability and robustness of the present results. One exception occurred wherein a fixed-effects model analysis identified a previously marginal PEP risk reduction associated with ulinastatin administrated both prior to and after ERCP (Fig. 2B) as being statistically significant ( $R R=0.60 ; 95 \%$ CI: $0.39-0.92 ; \mathrm{P}=0.02 ; \mathrm{I}^{2}=45$ ) (data no shown).

Effect of prophylactic ulinastatin administration on preventing PEHA. As presented in Table III, 9 studies including 875 patients receiving prophylactic ulinastatin and 1,281 patients receiving a placebo or no drug as a control were included in the meta-analysis on the efficacy of prophylactic ulinastatin in preventing PEHA $(4,6,8,9,11,13,14,16,17)$. The analysis revealed that prophylactic ulinastatin led to a significant PEHA risk reduction vs. the control ( $\mathrm{RR}=0.68$; 95\% CI: 0.56-0.83; $\mathrm{P}=0.0001$; $\mathrm{I}^{2}=19$ ); however, such significantly reduced PEHA risk only occurred when the ulinastatin administration began prior to ERCP (RR=0.62; 95\% CI: 0.50-0.77; P $<0.0001 ; \mathrm{I}^{2}=0$ ), but not during or after ERCP (Fig. 3A). Furthermore, post-ERCP ulinastatin administration in addition to the pre-ERCP administration appeared to be necessary to significantly reduce the PEHA risk (RR=0.65; 95\% CI: 0.51-0.84; $\mathrm{P}=0.00009 ; \mathrm{I}^{2}=0$; Fig. 3B).

Furthermore, a significantly reduced PEHA risk associated with ulinastatin vs. control was observed in patients with a low or average risk for PEP ( $R R=0.66$; 95\% CI: 0.54-0.82; $\left.\mathrm{P}=0.0001 ; \mathrm{I}^{2}=13\right)$, but not in high-risk patients $(\mathrm{RR}=0.71$; 95\% CI: 0.19-2.67; $\mathrm{P}=0.61 ; \mathrm{I}^{2}=89$; Fig. 3C). Ulinastatin was able to significantly reduce the risk of PEHA if administered at a high dose $(150,000$ or $200,000 \mathrm{U}$; RR=0.70; $95 \% \mathrm{CI}$ : $\left.0.58-0.85 ; \mathrm{P}=0.0004 ; \mathrm{I}^{2}=7\right)$, but not at a low dose $(100,000 \mathrm{U}$; Fig. 3D).

Furthermore sub-group analysis based on the quality of the studies revealed that the quality of the studies had an impact on the results: Although analysis of low-quality studies indicated a significant reduction of the risk of PEHA associated with ulinastatin vs. control, analysis of high- or average-quality studies only revealed a trend short of statistically significance of the reduced PEHA risk associated with ulinastatin (Fig. 3E).

In most analyses, using a fixed-effects model did not significantly impact the results obtained by using a random-effects model; however, there were a few exceptions. A fixed-effects model analysis indicated a statistically significant PEHA risk reduction in certain groups when the risk reductions for these groups were insignificant when analyzed using a random-effects model, including the cases with ulinastatin administration only prior to ERCP as well as high-risk patients, those receiving low-dosage ulinastatin and low-quality studies (data not shown), indicating the presence of substantial heterogeneities and inconsistencies among the studies included in these sub-groups. 


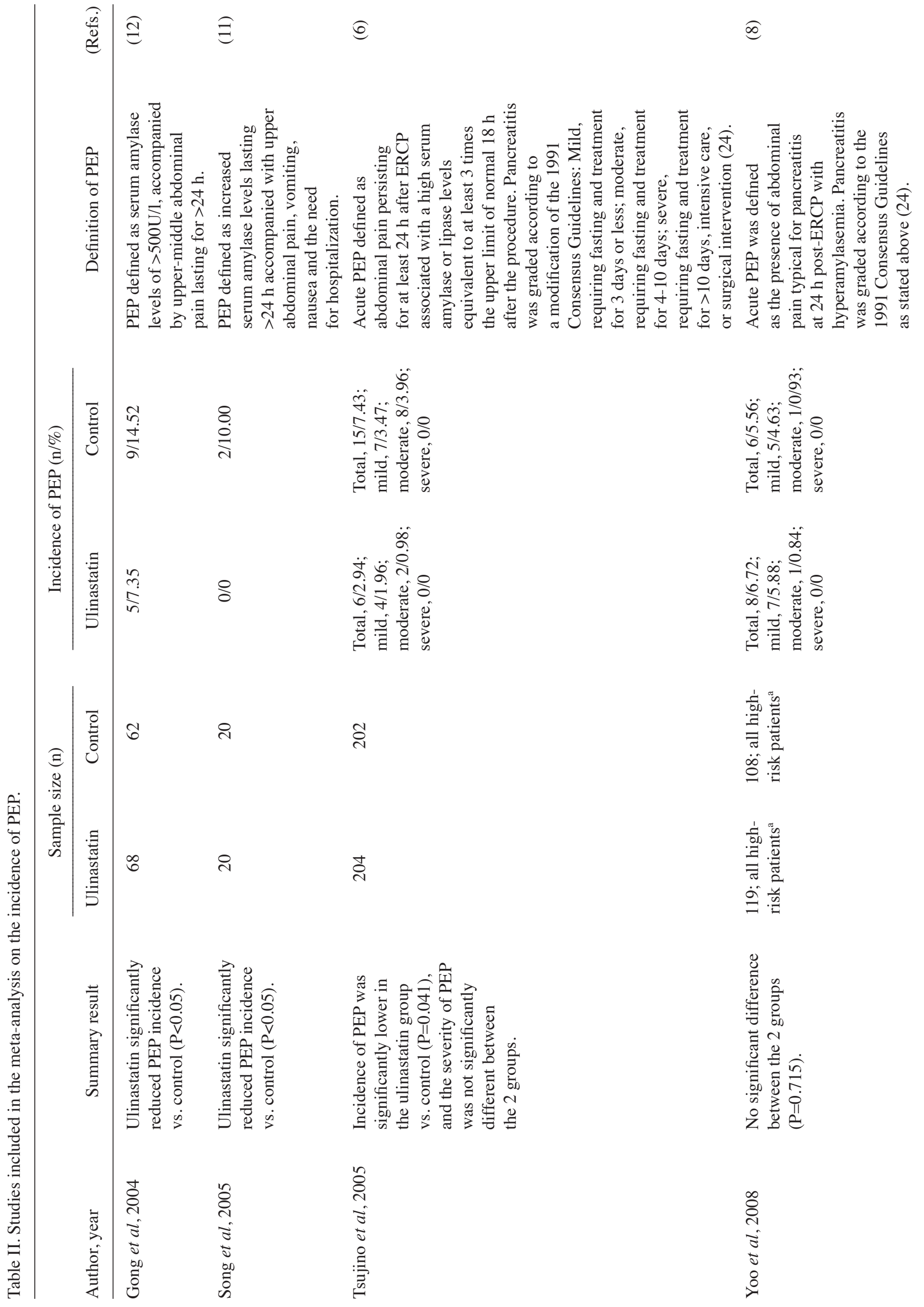




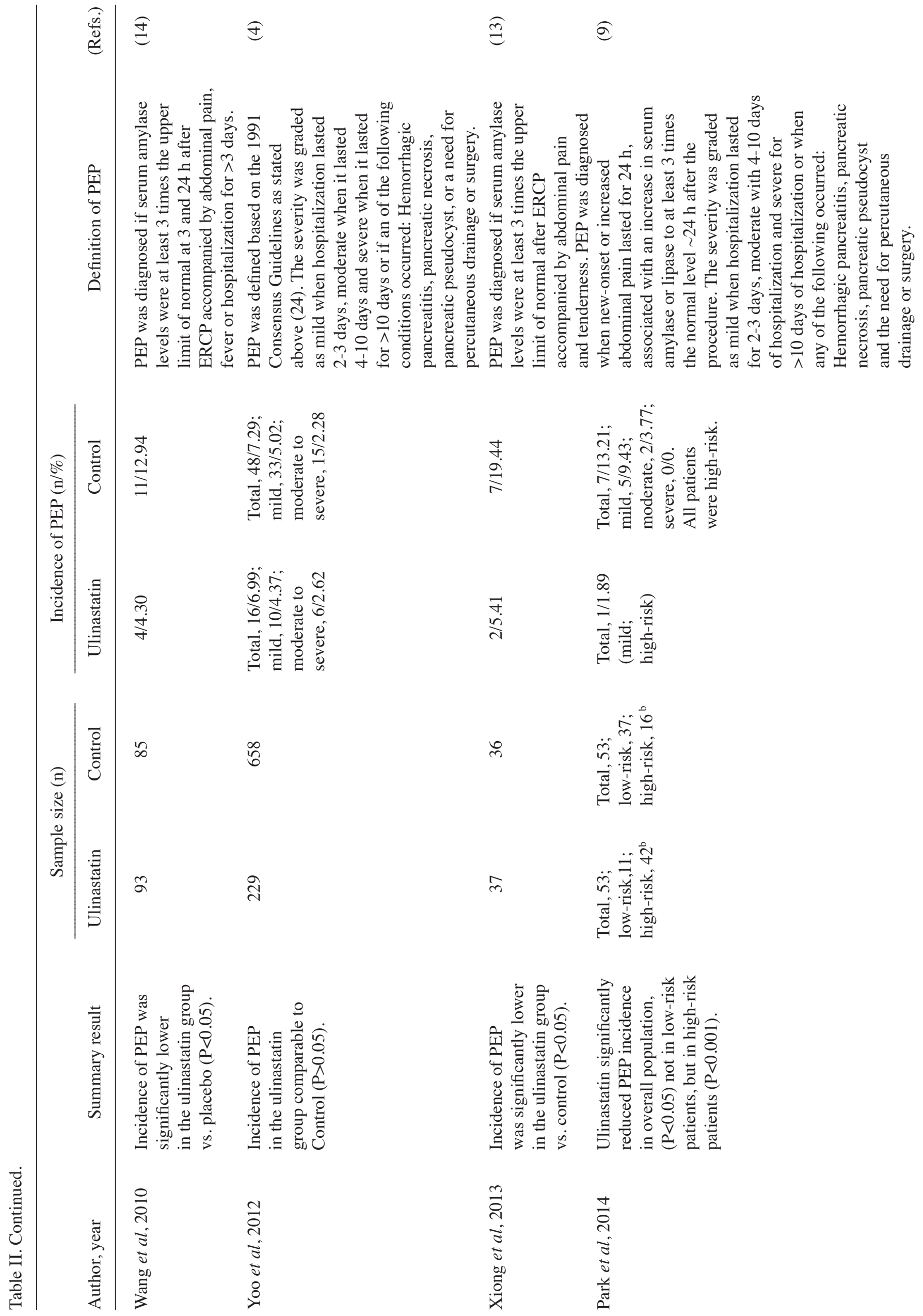




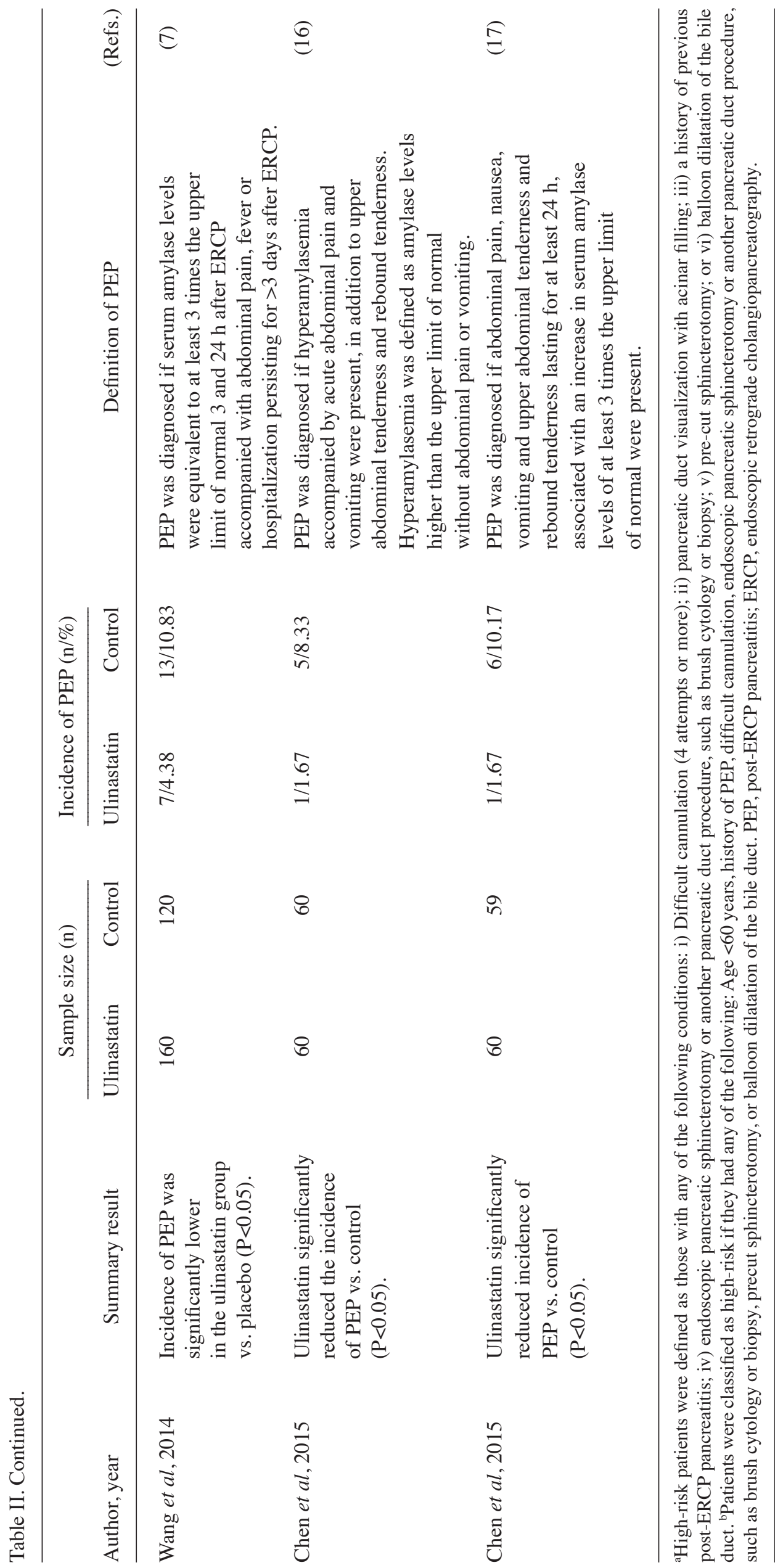







Effect of prophylactic ulinastatin administration on preventing post-ERCP abdominal pain. As presented in Table IV, 7 studies including 582 patients receiving prophylactic ulinastatin and 590 patients receiving placebo or nothing as controls were included in the meta-analysis on the efficacy of prophylactic ulinastatin administration in preventing post-ERCP abdominal pain $(6,8-10,15-17)$. The analysis revealed that overall, ulinastatin administration only marginally reduced the risk of post-ERCP abdominal pain vs. control ( $R R=0.67$; 95\% CI: 0.45-1.00; $\mathrm{P}=0.05 ; \mathrm{I}^{2}=67$ ), however, pre-ERCP ulinastatin administration significantly reduced the risk of post-ERCP abdominal pain ( $\mathrm{RR}=0.59$; 95\% CI: 0.42-0.83; $\mathrm{P}=0.002$; $\mathrm{I}^{2}=37$ ), while ulinastatin administration commenced during or after ERCP did not (Fig. 4A). Furthermore, post-ERCP ulinastatin administration in addition to the pre-ERCP administration appeared to be necessary to significantly reduce the incidence of post-ERCP abdominal pain $(\mathrm{RR}=0.56$; 95\% $\mathrm{CI}$ : 0.35-0.89; $\mathrm{P}=0.01 ; \mathrm{I}^{2}=54$; Fig. 4B).

In addition, patients with a low or average risk for PEP who received ulinastatin had a significantly reduced risk of post-ERCP abdominal pain vs. control $(\mathrm{RR}=0.59$; $95 \% \mathrm{CI}$ : $\left.0.42-0.81 ; \mathrm{P}=0.001 ; \mathrm{I}^{2}=20\right)$, but patients with a high risk for PEP did not (Fig. 4C). Furthermore, high-dose ulinastatin administration $(150,000 \mathrm{U})$ led to a significantly reduced incidence of post-ERCP abdominal pain ( $\mathrm{RR}=0.64 ; 95 \% \mathrm{CI}$ : $\left.0.44-0.93 ; \mathrm{P}=0.02 ; \mathrm{I}^{2}=0\right)$, while low-dosage ulinastatin administration did not (50,000 or 100,000 U; Fig. 4D).

The present sub-group analysis based on the quality of the studies revealed that the quality had an impact on the results. In fact, although analysis including low-quality studies revealed a significantly reduced incidence of post-ERCP abdominal pain associated with ulinastatin vs. control, analysis including high or average-quality studies did not (Fig. 4E).

Using a fixed-effects model, the marginally significant reduction of the risk of post-ERCP abdominal pain associated with ulinastatin reached statistical significance $(R R=0.71$; 95\% CI: 0.57-0.88; P=0.002; $\left.\mathrm{I}^{2}=67\right)$. Similar results were obtained for patients who received low-dosage ulinastatin and the sub-group only including low-quality studies, indicating heterogeneities and inconsistencies among these studies.

Heterogeneity and publication bias. Inter-study heterogeneities varied from none $\left(\mathrm{I}^{2}=0\right)$ to very high $\left(\mathrm{I}^{2}=96\right)$. Funnel plots for all of the analyses in the present study were slightly asymmetrical, wherein funnel plots for the analysis on the efficacy of prophylactic ulinastatin in preventing PEP were more symmetrical than those for the analysis on PEHA and post-ERCP abdominal pain (data not shown), indicating the possible presence of publication bias, with analysis on PEP having potentially less publication bias than those on PEHA and post-ERCP abdominal pain (data not shown).

\section{Discussion}

The present meta-analysis focused on the efficacy of prophylactic ulinastatin in preventing PEP, PEHA and post-ERCP abdominal pain. The analysis revealed that prophylactic ulinastatin significantly reduced the PEP risk vs. control; however, such significant risk reduction occurred only in patients with a low or average risk for PEP, with high-dosage ulinastatin 


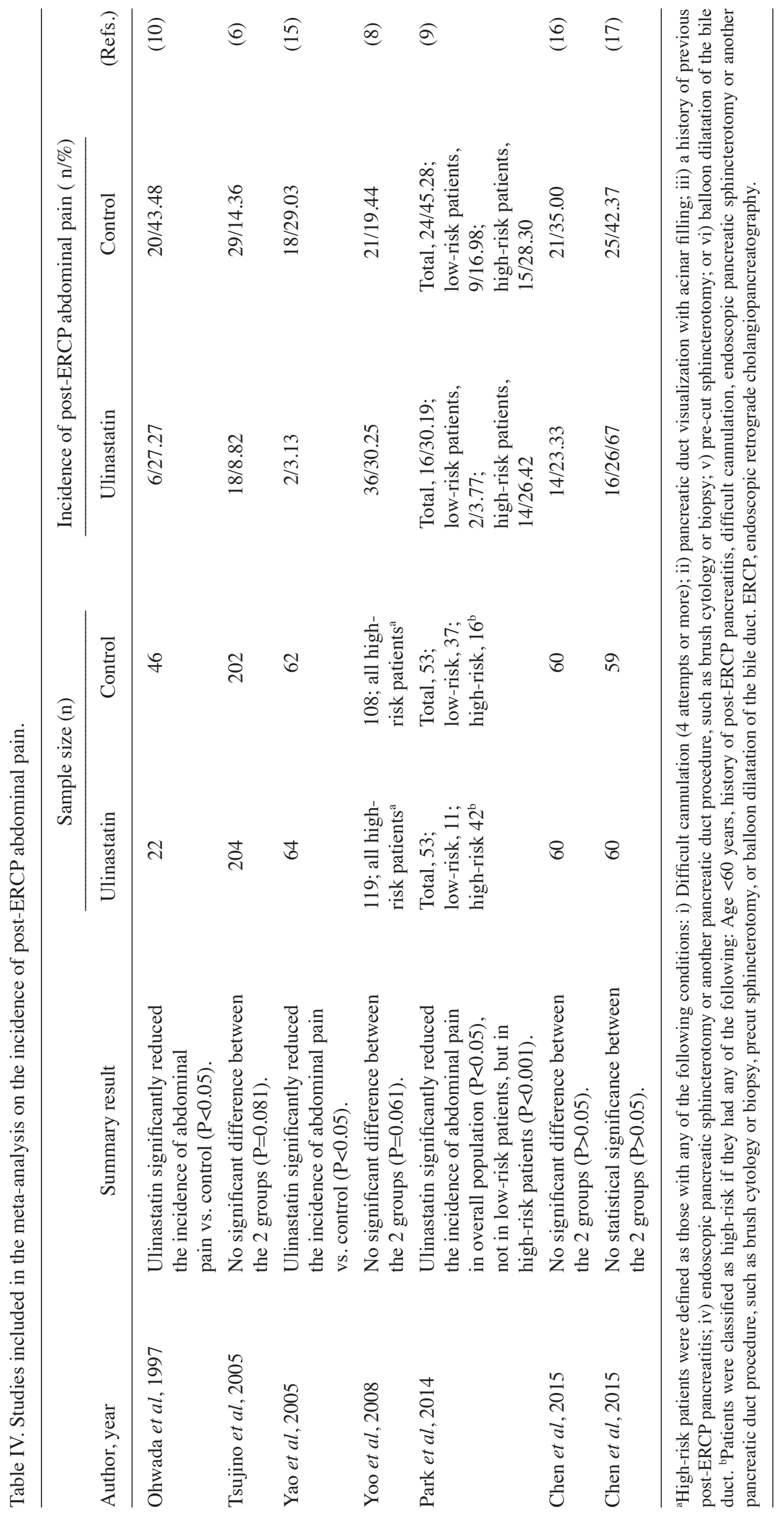




\begin{tabular}{|c|c|c|c|c|c|c|c|c|c|c|c|}
\hline A & \multicolumn{2}{|c|}{ Ulinastatin } & \multicolumn{2}{|c|}{ Control } & \multicolumn{3}{|c|}{ Risk Ratio } & \multicolumn{4}{|c|}{$\begin{array}{c}\text { Risk Ratio } \\
\text { M.H, Random, } 95 \% \mathrm{Cl}\end{array}$} \\
\hline \multicolumn{12}{|c|}{ 1.1.1 Ulinastatin administration began before ERCP } \\
\hline Gong et al & 5 & 68 & 9 & 62 & $11.0 \%$ & $0.51[0.18,1.43]$ & 2004 & & & - & \\
\hline Tsujino et al & 6 & 204 & 15 & 202 & $12.9 \%$ & $0.40[0.16,1.00]$ & 2005 & & & & \\
\hline Song et al & 0 & 20 & 2 & 20 & $1.8 \%$ & $0.20[0.01,3.92]$ & 2005 & & & & \\
\hline Yoo et al 2012 & 16 & 229 & 48 & 658 & $23.2 \%$ & $0.96[0.56,1.65]$ & 2012 & & & $\leftarrow$ & \\
\hline Park et al & 1 & 53 & 7 & 53 & $3.5 \%$ & $0.14[0.02,1.12]$ & 2014 & & & & \\
\hline Chen et al (Zhejiang JITCWM) & 1 & 60 & 5 & 60 & $3.3 \%$ & $0.20[0.02,1.66]$ & 2015 & & & & \\
\hline $\begin{array}{l}\text { Chen et al (Chinese Rural Medicine) } \\
\text { Subtotal (95\% CI) }\end{array}$ & 1 & $\begin{array}{r}60 \\
694\end{array}$ & 6 & $\begin{array}{r}59 \\
1114\end{array}$ & $\begin{array}{r}3.4 \% \\
59.1 \%\end{array}$ & $\begin{array}{l}0.16[0.02,1.32] \\
0.46[0.26,0.83]\end{array}$ & 2015 & & & & \\
\hline Total events & 30 & & 92 & & & & & & & & \\
\hline \multicolumn{12}{|c|}{$\begin{array}{l}\text { Heterogeneity: } \mathrm{Tau}^{2}=0.18 ; \mathrm{Ch}^{2}=8.78, \mathrm{df}=6(P=0.19) ; \mathrm{F}^{2}=32 \% \\
\text { Test for overall effect } Z=2.59(P=0.010)\end{array}$} \\
\hline \multicolumn{12}{|c|}{ 1.1.2 Ulinastatin administration begain during ERCP (in contrast medium) } \\
\hline Wang et al & 4 & 93 & 11 & 85 & $10.0 \%$ & $0.33[0.11,1.00]$ & 2010 & & & & \\
\hline $\begin{array}{l}\text { Wang et al } 2014 \\
\text { Subtotal }(95 \% \mathrm{Cl})\end{array}$ & 7 & $\begin{array}{l}160 \\
253\end{array}$ & 13 & $\begin{array}{l}120 \\
205\end{array}$ & $\begin{array}{l}13.6 \% \\
23.6 \%\end{array}$ & $\begin{array}{l}0.40[0.17,0.98] \\
0.37[0.19,0.75]\end{array}$ & 2014 & & & & \\
\hline Total events & 11 & & 24 & & & & & & & & \\
\hline \multicolumn{12}{|c|}{$\begin{array}{l}\text { Heterogeneity. } \text { Tau }^{2}=0.00 ; \mathrm{Ch}^{2}=0.07, \mathrm{df}=1(\mathrm{P}=0.79) ; \mathrm{P}^{2}=0 \% \\
\text { Test for overall effect. } Z=2.78(P=0.005)\end{array}$} \\
\hline \multicolumn{12}{|c|}{ 1.1.3 Ulinastatin administration began after ERCP } \\
\hline Yoo et al & 8 & 119 & 6 & 108 & $11.2 \%$ & $1.21[0.43,3.38]$ & 2008 & & & & \\
\hline $\begin{array}{l}\text { Xiong et al } \\
\text { Subtotal }(95 \% \mathrm{Cl})\end{array}$ & 2 & $\begin{array}{r}37 \\
156\end{array}$ & 7 & $\begin{array}{r}36 \\
144\end{array}$ & $\begin{array}{r}6.1 \% \\
17.3 \%\end{array}$ & $\begin{array}{l}0.28[0.06,1.25] \\
0.64[0.15,2.70]\end{array}$ & 2013 & & & & \\
\hline \multirow{2}{*}{\multicolumn{12}{|c|}{$\begin{array}{l}\text { Heterogeneity: } \text { Tau }^{2}=0.66 ; \mathrm{Ch}^{2}=2.53 \text {, df }=1(P=0.11) ; \mathrm{F}^{\mathrm{P}}=60 \% \\
\text { Test for overall effect: } Z=0.60(P=0.55)\end{array}$}} \\
\hline & & & & & & & & & & & \\
\hline Total $(95 \% \mathrm{Cl})$ & & 1103 & & 1463 & $100.0 \%$ & $0.49[0.33,0.74]$ & & & & & \\
\hline Total events & 51 & & 129 & & & & & & & & \\
\hline $\begin{array}{l}\text { Heterogeneity: } \operatorname{Tau}^{2}=0.10 ; \mathrm{Ch}^{2}=13.1 \\
\text { Test for owerall effect. } Z=3.44(P=0.0 \\
\text { Test for subaroun differences: } \mathrm{ChP}^{2}=\mathrm{C}\end{array}$ & $\begin{array}{l}9, \mathrm{df}=10 \\
06) \\
.51, \mathrm{df}=2\end{array}$ & $\begin{array}{l}(P=0.2 \\
(P=0.7\end{array}$ & 1): $; I^{2}=24$ & & & & & 0.01 & $\begin{array}{c}0.1 \\
\text { Favours ullina }\end{array}$ & Favours control & 100 \\
\hline B & Ulinast & & Contr & & & Risk Ratio & & & Risk R & Ratio & \\
\hline Study or Subgroup & Events & Total & Events & Total & Weight & M-H, Random, $95 \% \mathrm{Cl}$ & Year & & M-H, Randon & $\mathrm{om}, 95 \% \mathrm{Cl}$ & \\
\hline 1.2.1 Ulinastatin only administered $b$ & fore ERCI & & & & & & & & & & \\
\hline Song et al & 0 & 20 & 2 & 20 & $3.6 \%$ & $0.20[0.01,3.92]$ & 2005 & & & & \\
\hline $\begin{array}{l}\text { Tsujino et al } \\
\text { Subtotal }(95 \% \mathrm{Cl})\end{array}$ & 6 & $\begin{array}{l}204 \\
224\end{array}$ & 15 & $\begin{array}{l}202 \\
222\end{array}$ & $\begin{array}{l}22.1 \% \\
25.7 \%\end{array}$ & $\begin{array}{l}0.40[0.16,1.00] \\
0.37[0.15,0.90]\end{array}$ & 2005 & & & & \\
\hline Total events & 6 & & 17 & & & & & & & & \\
\hline $\begin{array}{l}\text { Heterogeneity } \text { Tau }^{2}=0.00 ; \mathrm{Ch}^{2}=0.1 \\
\text { Test for overall effect. } Z=2.19(\mathrm{P}=0 . \mathrm{l}\end{array}$ & 1) $d f=1(P$ & $=0.67$. & ${ }^{2}=0 \%$ & & & & & & & & \\
\hline 1.2.2 Ulinastatin administered before & and after & ERCP & & & & & & & & & \\
\hline Gong et al & 5 & 68 & 9 & 62 & $19.4 \%$ & $0.51[0.18,1.43]$ & 2004 & & & - & \\
\hline Yoo et al 2012 & 16 & 229 & 48 & 658 & $34.7 \%$ & $0.96[0.56,1.65]$ & 2012 & & & & \\
\hline Park et al & 1 & 53 & 7 & 53 & $6.9 \%$ & $0.14[0.02,1.12]$ & 2014 & & & & \\
\hline Chen et al (Zhejiang JITCWM) & 1 & 60 & 5 & 60 & $6.6 \%$ & $0.20[0.02,1.66 \mid$ & 2015 & & & - & \\
\hline $\begin{array}{l}\text { Chen et al (Chinese Rural Medicine) } \\
\text { Subtotal (95\% Cl) }\end{array}$ & 1 & $\begin{array}{r}60 \\
470\end{array}$ & 6 & $\begin{array}{r}59 \\
892\end{array}$ & $\begin{array}{r}6.8 \% \\
74.3 \%\end{array}$ & $\begin{array}{l}0.16[0.02,1.32] \\
0.45[0.20,1.00]\end{array}$ & 2015 & & & & \\
\hline Total events & 24 & & 75 & & & & & & & & \\
\hline $\begin{array}{l}\text { Heterogeneity } \text { Tau }^{2}=0.34 ; \mathrm{Ch}^{2}=7.2 \\
\text { Test for overall effect. } Z=1.97(\mathrm{P}=0 . \mathrm{l}\end{array}$ & (f) $d f=4(P$ & $=0.12)$ & $z^{2}=45 \%$ & & & & & & & & \\
\hline Total (95\% Cl) & & 694 & & 1114 & $100.0 \%$ & $0.46[0.26,0.83]$ & & & & & \\
\hline Total events & 30 & & 92 & & & & & & & & \\
\hline $\begin{array}{l}\text { Heterogeneity } \mathrm{Tau}^{2}=0.18 ; \mathrm{Ch}^{2}=8.7 \\
\text { Test for overall effect. } Z=2.59(\mathrm{P}=0 . \mathrm{C} \\
\text { Test for subqroup differences: } \mathrm{Chi}^{2}=\end{array}$ & $\begin{array}{l}d f=6(P \\
10) \\
09 . \\
0 f t=1\end{array}$ & $\begin{array}{l}=0.19) \\
(P=0\end{array}$ & $\begin{array}{l}F^{2}=32 \% \\
76), 1^{2}=0\end{array}$ & & & & & 0.01 & $\begin{array}{c}0.1 \\
\text { Favours ulinastatin }\end{array}$ & $\begin{array}{c}10 \\
\text { Favours control }\end{array}$ & 100 \\
\hline 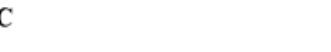 & Ulinaste & & Contre & & & Risk Ratio & & & & Ratio & \\
\hline Study or Subgroup & Events & Total & Events & Total & Weight & M-H, Random, $95 \% \mathrm{Cl}$ & Year & & M-H, Rando & dom, $95 \% \mathrm{Cl}$ & \\
\hline 1.3.1 High-risk patients & & & & & & & & & & & \\
\hline Yoo et al & 8 & 119 & 6 & 108 & $11.4 \%$ & $1.21[0.43,3.38]$ & 2008 & & & & \\
\hline $\begin{array}{l}\text { Park et al - High-risk patients } \\
\text { Subtotal }(95 \% \mathrm{Cl})\end{array}$ & 1 & $\begin{array}{r}42 \\
161\end{array}$ & 7 & $\begin{array}{r}16 \\
124\end{array}$ & $\begin{array}{r}4.4 \% \\
15.8 \%\end{array}$ & $\begin{array}{l}0.05[0.01,0.41] \\
0.29[0.01,6.37]\end{array}$ & 2014 & & & & \\
\hline Total events & 9 & & 13 & & & & & & & & \\
\hline $\begin{array}{l}\text { Heterogeneily: Tau }=4.34 ; \mathrm{Ch}^{z}=7.52 \\
\text { Test for overall effect } Z=0.79(P=0.4\end{array}$ & $d r=1(P=$ & $=0.006$ & $F=879$ & & & & & & & & \\
\hline 1.3.2 Other patients & & & & & & & & & & & \\
\hline Gong et al & 5 & 68 & 9 & 62 & $11.3 \%$ & $0.51[0.18,1.43]$ & 2004 & & & & \\
\hline Tsujino et al & 8 & 204 & 15 & 202 & $12.7 \%$ & $0.40[0.16,1.00]$ & 2005 & & & & \\
\hline Song et al & 0 & 20 & 2 & 20 & $2.2 \%$ & $0.20[0.01,3.92]$ & 2005 & & & & \\
\hline Wang et al & 4 & 93 & 11 & 85 & $10.5 \%$ & $0.33[0.11,1.00]$ & 2010 & & & & \\
\hline Yoo et al 2012 & 16 & 229 & 48 & 658 & $19.0 \%$ & $0.96[0.56,1.65]$ & 2012 & & & & \\
\hline $\begin{array}{l}\text { Xiong et al } \\
\text { Wang et al } 2014\end{array}$ & $\begin{array}{l}2 \\
7\end{array}$ & $\begin{array}{r}37 \\
160\end{array}$ & $\begin{array}{r}7 \\
13\end{array}$ & $\begin{array}{r}36 \\
120\end{array}$ & $\begin{array}{r}7.0 \% \\
13.3 \%\end{array}$ & $\begin{array}{l}0.28[0.06,1.25] \\
0.40[0.17,0.98]\end{array}$ & $\begin{array}{l}2013 \\
2014\end{array}$ & & & & \\
\hline Park et al - LoW-risk patients & 0 & 11 & 0 & 37 & & Not estimable & 2014 & & & & \\
\hline Chen et al (Chinese Rural Medicine) & 1 & 60 & 6 & 59 & $4.2 \%$ & $0.16[0.02,1.32]$ & 2015 & & & - & \\
\hline $\begin{array}{l}\text { Chen et al (Zhejiang JITCWM) } \\
\text { Subtotal ( } 95 \% \text { CI) }\end{array}$ & 1 & $\begin{array}{r}60 \\
942\end{array}$ & 5 & $\begin{array}{r}60 \\
1339\end{array}$ & $\begin{array}{r}4.1 \% \\
84.2 \%\end{array}$ & $\begin{array}{l}0.20[0.02,1.66] \\
0.49[0.33,0.72]\end{array}$ & 2015 & & & & \\
\hline $\begin{array}{l}\text { Subtotal }(95 \% \text { CI }) \\
\text { Total events }\end{array}$ & 42 & 942 & 116 & & $84.2 \%$ & $0.49[0.33,0.72]$ & & & & & \\
\hline $\begin{array}{l}\text { Heterogeneity. Tau }=0.05 ; \mathrm{Chi}^{2}=9.1 \\
\text { Test for overall effect } Z=3.59(P=0.0\end{array}$ & $\begin{array}{l}\mathrm{df}=8(\mathrm{P}= \\
03)\end{array}$ & $=0.33) ;$ & $I^{2}=13 \%$ & & & & & & & & \\
\hline Total (95\% Cl) & & 1103 & & 1463 & $100.0 \%$ & $0.44[0.28,0.71]$ & & & & & \\
\hline Total events & 51 & & 129 & & & & & & & & \\
\hline $\begin{array}{l}\text { Heterogeneity. } \text { Tau }^{2}=0.22 ; \mathrm{Chi}^{2}=16 . \\
\text { Test for overall effect } Z=3.42(P=0.0 \\
\text { Testfor subaroun differences: } \mathrm{Ch}^{2}=\end{array}$ & b6) $d f=10$ & $P=0.0$ & 8); $1^{2}=40$ & & & & & & $\begin{array}{c}0.1 \\
\text { Favours ulinastatin }\end{array}$ & $\begin{array}{c}10 \\
\text { contro }\end{array}$ & 100 \\
\hline
\end{tabular}

Figure 2. Incidence of PEP in patients who received prophylactic ulinastatin vs. control. (A) Analysis with stratification by whether the ulinastatin administration began prior to, during or after ERCP. (B) Analysis for patients who began ulinastatin administration prior to ERCP, further stratified by whether ulinastatin was administered only prior to ERCP or also after ERCP. (C) Analysis with patients stratified into those with high risk for PEP and others. 


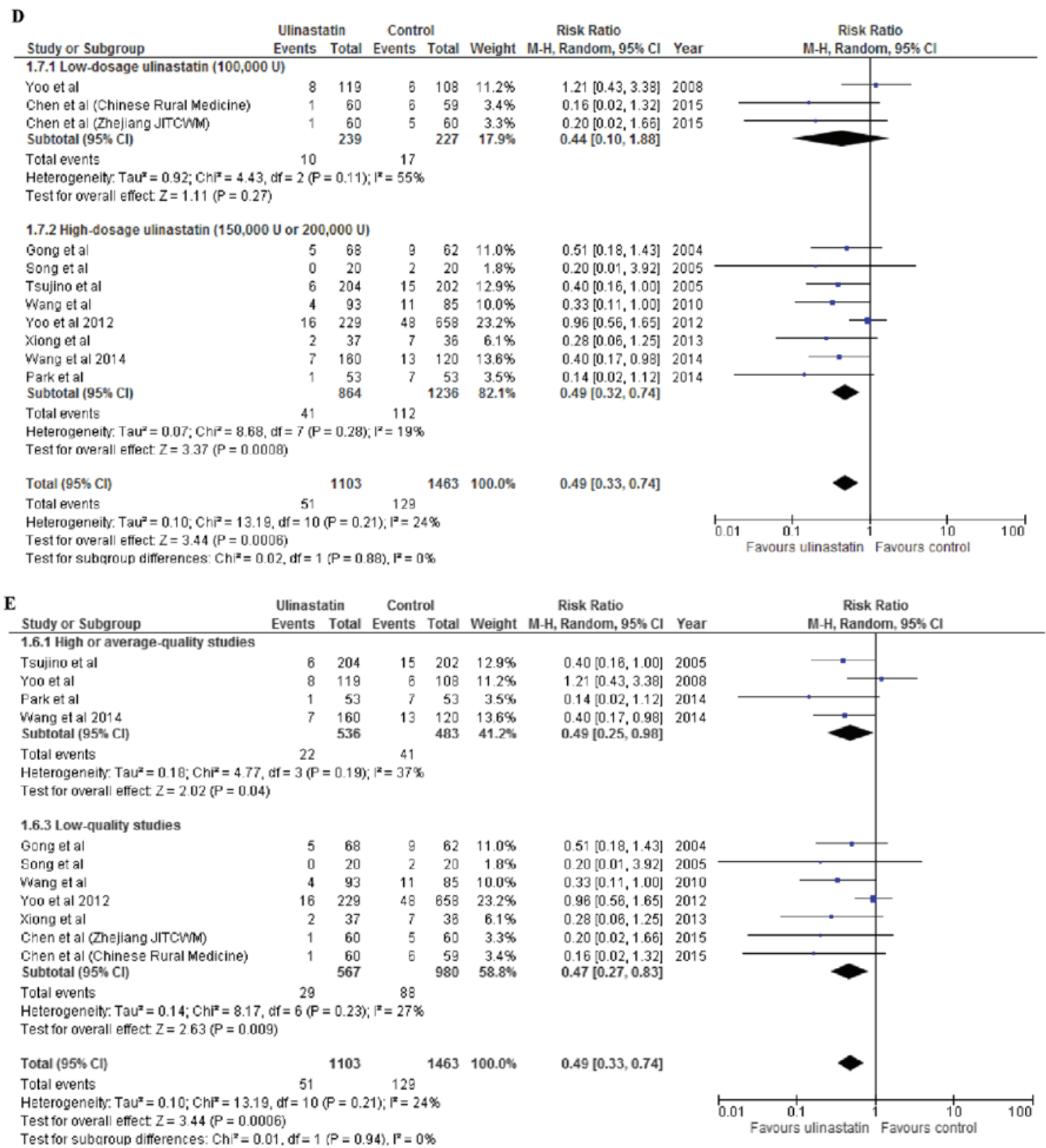

Figure 2. Continued. (D) Analysis with stratification by the dosage of ulinastatin administered: Low-dosage group (100,000 U) and high-dosage group $(150,000$ or $200,000 \mathrm{U})$. (E) Analysis stratified by the qualities of the included studies: High or average-quality studies (Jadad score, $\geq 2)$ and low-quality studies (Jadad score, <2). The squares and horizontal lines represent risk ratio and 95\% CI for included studies, respectively. CI, confidence interval; M-H, Mantel-Haenszel; ERCP, endoscopic retrograde cholangiopancreatography; PEP, post-ERCP pancreatitis; df, degrees of freedom.

$(150,000$ or $200,000 \mathrm{U})$ and when the ulinastatin administration began prior to or during ERCP. Additional post-ERCP ulinastatin administration was unnecessary. Prophylactic ulinastatin administration also significantly reduced the risk of PEHA and post-ERCP abdominal pain in patients with a low or average risk for PEP, with high-dosage ulinastatin administration $(150,000$ or $200,000 \mathrm{U})$, and when ulinastatin administration began prior to ERCP with the help of additional post-ERCP ulinastatin administration.

The present results on the preventive effect of ulinastatin on PEP, PEHA and post-ERCP abdominal pain were rather consistent with a couple of exceptions. Ulinastatin significantly reduced the risk of PEP if administration began either prior to or during ERCP, and pre-ERCP administration alone was sufficient. However, to effectively prevent PEHA or abdominal pain, administration was required to start prior to ERCP with additional post-ERCP ulinastatin administration. Such differences may indeed be true, since although PEHA and post-ERCP abdominal pain are early indicators of possible PEP, these 3 are not the same. However, the small number of studies included in each sub-group of these analyses may have been responsible for differences obtained. More relevant studies are required to further explore these topics.

The present results on PEP and PEHA were consistent with those of the meta-analysis published by Chen et al (3) in 2010, which included 5 randomized controlled studies and found that ulinastatin prevented PEP and PEHA in average-risk patients when administered intravenously at a dose of $\geq 150,000 \mathrm{U}$. However, the results of the present study were inconsistent with the meta-analysis by Yuhara et al (1) from 2014, including 
A

Study or Subgroup

Ulinastatin Control Risk Ratio Events Total Events Total Weight M.H, Random, $95 \% \mathrm{Cl}$ Year 2.1.1 Ulinastatin administration began before ERCP

Tsujino et al

Song et al

Yoo et al 2012

Parket al

Chen et al (Chinese Rural Medicine)

Chen et al (Zhejiang JITCWM)

Subtotal $(95 \% \mathrm{Cl})$

Total events
Heterogeneity $T_{a} u^{z}=0.00 ; C_{i} i^{z}=4.49, \quad$ of $=5(P=0.48) ; F^{z}=0 \%$

$\begin{array}{lllll}30 & 204 & 50 & 202 & 16.7 \%\end{array}$

$0.591039,0.0912005$

$0.22[0.05,0.90] 2005$

$0.76[0.50,1.16] 2012$

$0.70[0.47,1.05] 2014$

$0.70[0.47,1.05] 2014$
$0.47[0.25,0.88] 2015$

$0.50[0.23,1.08] 2015$

$0.62[0.50,0.77]$

Test for overall effect: $Z=4.42(P<0.0001)$

2.1.2 Ulinastatin administration began during ERCP

\begin{tabular}{|c|c|c|c|c|c|c|}
\hline Wang et al & 36 & 93 & 38 & & & $0.87[0.61,1.23]$ \\
\hline Subtotal $(95 \% \mathrm{Cl})$ & & 93 & & 85 & $20.8 \%$ & $0.87[0.61,1.23]$ \\
\hline Total events & 36 & & 38 & & & \\
\hline
\end{tabular}

Total events

$36 \quad 38$

$9465816.4 \%$

$30 \quad 53 \quad 16.8 \%$

$\begin{array}{lll}16 & 60 & 5.9 \%\end{array}$

Test for overall effect: $Z=0.81(P=0.42)$

2.1.3 Ulinastatin administration began after ERCP

$\begin{array}{lrrrrrrr}\text { Yoo et al } & 13 & 119 & 9 & 108 & 5.4 \% & 1.31[0.58,2.94] & 2008 \\ \text { Xlong et al } & 9 & 37 & 16 & 36 & 7.5 \% & 0.55[0.28,1.08] & 2013\end{array}$

Subtotal $(95 \% \mathrm{Cl}$

Total euents

Test for owerall effect: $Z=0.44(P=0.66)$

$\left.\begin{array}{lllll}\text { Total }(95 \% \mathrm{Cl}) & 875 & 1281 & 100.0 \% & 0.68\end{array}\right]$

$\begin{array}{lcc}\text { Total events } & 155 & 285 \\ \text { Heterogeneity. } \text { Tau }^{2}=0.02 ; \mathrm{Chi}^{2}=9.94, \mathrm{df}=8(\mathrm{P}=0.27) ; \mathrm{F}^{\mathrm{N}}=19 \%\end{array}$

Test for averall effect $Z=3.83(P=0.0001)$

Test for subaroun differences: $\mathrm{Chr}^{2}=2.71$, $\mathrm{df}=2(\mathrm{P}=0.26) \cdot \mathrm{I}^{2}=26.1$ \% $0.82[0.35,1.95]$

B

Ulinastatin Control

Risk Ratio M. H, Random, $95 \% \mathrm{Cl}$

\begin{tabular}{|c|c|c|c|c|c|c|c|}
\hline Study or Subgroup & Events & Total & Events & Total & Weight & M-H, Random, $95 \% \mathrm{Cl}$ & \\
\hline \multicolumn{8}{|c|}{ 2.2.1 Ulinastatin only administered before ERCP } \\
\hline Song et al & 2 & 20 & 9 & 20 & $2.3 \%$ & $0.22[0.05,0.90]$ & \\
\hline $\begin{array}{l}\text { Tsujino et al } \\
\text { Subtotal }(95 \% \mathrm{Cl})\end{array}$ & 30 & $\begin{array}{l}204 \\
224\end{array}$ & 50 & $\begin{array}{l}202 \\
222\end{array}$ & $\begin{array}{l}26.5 \% \\
28.7 \%\end{array}$ & $\begin{array}{l}0.59[0.39,0.89] \\
0.46[0.20,1.07]\end{array}$ & \\
\hline Total events & 32 & & 59 & & & & \\
\hline \multicolumn{8}{|c|}{$\begin{array}{l}\text { Heterogeneity: Tau }=0.21 ; \mathrm{Chi}^{2}=1.76, \mathrm{df}=1(\mathrm{P}=0.19) ; \mathrm{P}=43 \% \\
\text { Test for owerall effect } \mathrm{Z}=1.79(\mathrm{P}=0.07)\end{array}$} \\
\hline \multicolumn{8}{|c|}{ 2.2.2 Ulinastatin administered both before and after ERCP } \\
\hline Yoo et al 2012 & 25 & 229 & 94 & 658 & $25.7 \%$ & $0.76[0.50,1.16]$ & \\
\hline Parket al & 21 & 53 & 30 & 53 & $26.6 \%$ & $0.70[0.47,1.05]$ & \\
\hline Chen et al (Chinese Rural Medicin & 11 & 60 & 23 & 59 & $11.4 \%$ & $0.47[0.25,0.88]$ & \\
\hline $\begin{array}{l}\text { Chen et al (Zhejiang JITCMM) } \\
\text { Subtotal ( } 95 \% \mathrm{Cl})\end{array}$ & 8 & $\begin{array}{r}60 \\
402\end{array}$ & 16 & $\begin{array}{r}60 \\
830\end{array}$ & $\begin{array}{r}7.5 \% \\
71.3 \%\end{array}$ & $\begin{array}{l}0.50[0.23,1.08] \\
0.65[0.51,0.84]\end{array}$ & \\
\hline
\end{tabular}

Total events $\quad 65 \quad 163$

Heterogeneity: $\operatorname{Tau}^{2}=0.00 ; \mathrm{Chi}^{2}=2.20, \mathrm{df}=3(P=0.53) ; \mathrm{P}=0 \%$

Test for overall effect $Z=3.34(P=0.0009)$

$\begin{array}{lllll}\text { Total }(95 \% \mathrm{Cl}) & 626 & 1052 & 100.0 \% & 0.62[0.50,0.77]\end{array}$

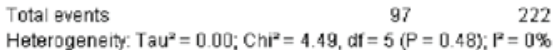

Test for overall effect $Z=4.42(P=0.0001)$

Test for subaroun differences: $C h \mathbf{P}^{2}=0.61, d f=\left.1(P=0.43)^{2}\right|^{2}=0 \%$

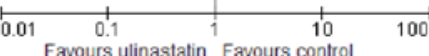

C

\begin{tabular}{|c|c|c|c|c|c|c|}
\hline \multirow[b]{2}{*}{ Study or Subgroup } & \multicolumn{2}{|c|}{ Ulinastatin } & \multicolumn{2}{|c|}{ Control } & \multirow[b]{2}{*}{ Weight } & Risk Ratio \\
\hline & Events & Total & Events & Total & & M-H, Random, $95 \% \mathrm{Cl}$ \\
\hline 2.3.1 High-risk patients & & & & & & \\
\hline Yoo et al & 13 & 119 & g & 108 & $6.5 \%$ & $1.31[0.58,2.94]$ \\
\hline $\begin{array}{l}\text { Park et al - High-risk patients } \\
\text { Subtotal }(95 \% \mathrm{Cl})\end{array}$ & 17 & $\begin{array}{r}42 \\
161\end{array}$ & 16 & $\begin{array}{r}16 \\
124\end{array}$ & $\begin{array}{l}15.5 \% \\
22.1 \%\end{array}$ & $\begin{array}{l}0.42[0.29,0.61] \\
0.71[0.19,2.67]\end{array}$ \\
\hline
\end{tabular}

Total events $30 \quad 25$

Heterogeneity: Tau $^{2}=0.81 ; \mathrm{Ch}^{2}=8.90$, df
Test for overall effect $Z=0.51(P=0.81)$

2.3.2 Other patients

Song et al

Tsujino et al

Wang et al

Yoo et al 2012

Xiong et al

Park et al - Low-risk patients

Chen et al (Zhejiang JITCWM)

Chen et al (Chinese Rural Medicine)

Subtotal $(95 \% \mathrm{Cl})$

Total events
Heterogeneity: $\mathrm{Tau}^{z}=0.01 ; \mathrm{Ch}^{\mathbf{z}}=8.05, \mathrm{df}=7(\mathrm{P}=0.33) ; \mathrm{F}^{\mathbf{z}}=13 \%$

Test for overall effect $Z=3.84(P=0.0001)$

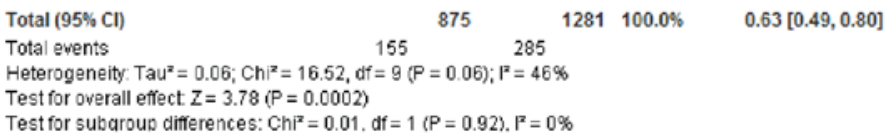

$0.22[0.05,0.90] 2005$ $0.59[0.39,0.89] 2005$ $0.87[0.61,1.23] 2010$ $0.76[0.50,116] 2012$ $0.55[0.28,1.08] 2013$ $0.50[0.23,1.08] 2015$ $0.47[0.25,0.88] 2015$ $0.66[0.54,0.82]$

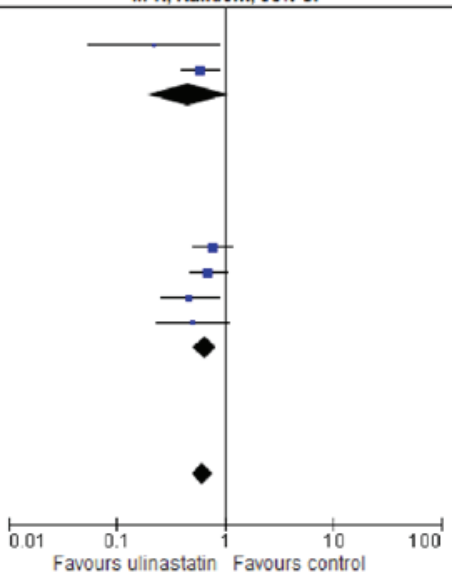

Risk Ratio M-H, Random, $95 \% \mathrm{Cl}$

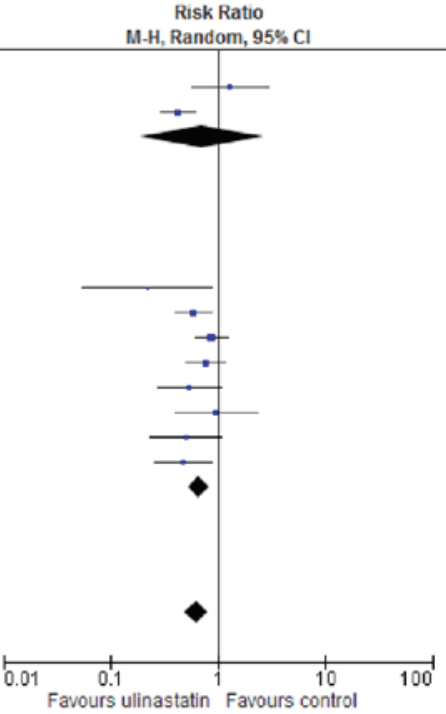

Figure 3. Incidence of post-ERCP hyperamylasemia in patients who received prophylactic ulinastatin vs. control. (A) Analysis stratified by whether ulinastatin administration began prior to, during or after ERCP. (B) Analysis for patients who began ulinastatin administration prior to ERCP, further stratified by whether ulinastatin was administered only prior to or also after ERCP. (C) Analysis with patients stratified into those with high risk for post-ERCP hyperamylasemia and other patients. 
D

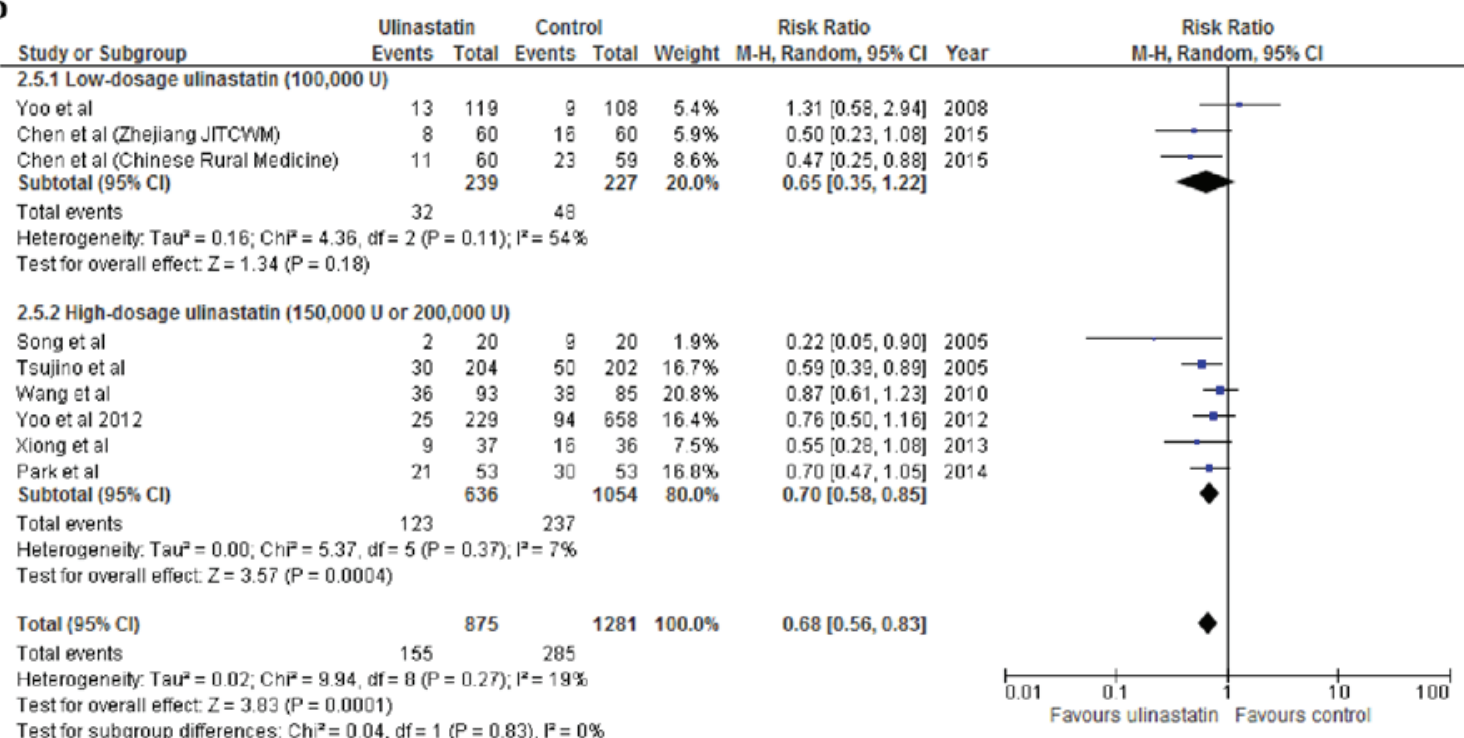

$\mathbf{E}$

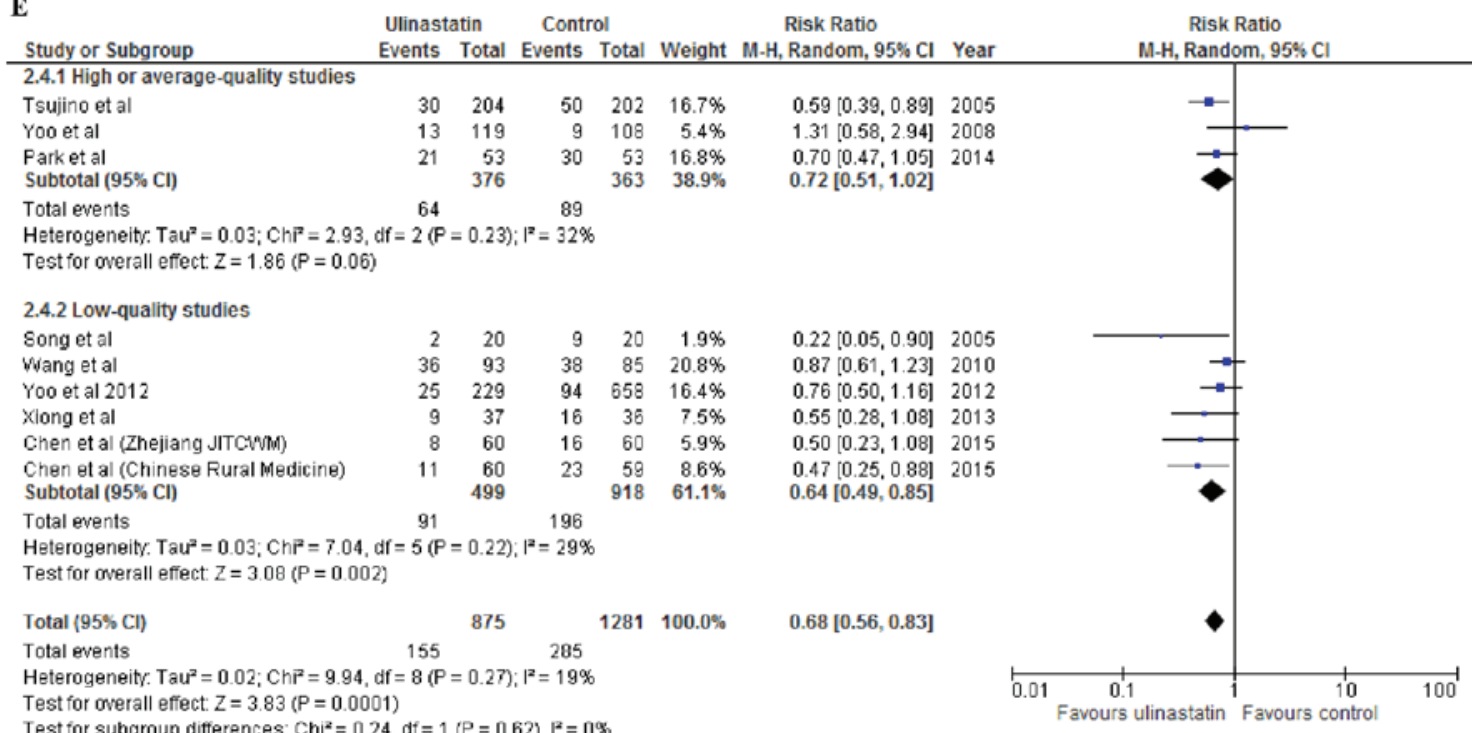

Figure 3. Continued. (D) Analysis stratified by the dosage of ulinastatin administered: Low-dosage group (100,000 U) and high-dosage group (150,000 or $200,000 \mathrm{U}$ ). (E) Analysis stratified by the qualities of the included studies: High or average-quality studies (Jadad score, $\geq 2$ ) and low-quality studies (Jadad score, $<2$ ). The squares and horizontal lines represent risk ratio and 95\% CI for included studies, respectively. CI, confidence interval; M-H, Mantel-Haenszel; ERCP, endoscopic retrograde cholangiopancreatography; df, degrees of freedom.

6 randomized trials, two of which were high-quality studies with a Jadad score $\geq 3$, which found that ulinastatin was not associated with a decreased PEP risk regardless of the quality of the studies. The present meta-analysis with 13 studies and a larger population size confirmed and further expanded on the findings by Chen et al (3). Since the present meta-analysis included several studies published after the study of Yuhara et al (1) and also several earlier-published Chinese studies not included by them, and since these studies were grouped into high- or average-quality (Jadad score, $\geq 2$ ) and low-quality studies (Jadad score, $<2$ ) in the present study, it is no surprise that the results are different. Considering that the present analysis incorporated the latest published studies and also several relevant Chinese studies not included in the study by Yuhara et al (1), and is thus more comprehensive, the results may be more robust. In addition, the preventive effect of prophylactic ulinastatin on post-ERCP abdominal pain was further analyzed. When there is no pain, there is no pancreatitis, and PEP only occurs in patients who complain of abdominal pain accompanied by hyperamylasemia $(3,22)$. Therefore, similar to PEHA, the effect of prophylactic ulinastatin on post-ERCP abdominal pain should also be analyzed.

PEP is the most common complication of ERCP and is potentially fatal. It starts with premature intra-acinar trypsin activation and subsequent activation of various proteolytic enzymes (6). Trypsin activates enzymes such as phopholipase $\mathrm{A}_{2}$, kallikrein and elastase, and leads to injury of acinar cells, autodigestion and a systemic inflammatory response (6). Given that trypsin activation is the triggering event, ulinastatin, a trypsin inhibitor, makes a perfectly plausible candidate for PEP prevention. In addition, ulinastatin has the added advantage of being a strong inhibitor of a wide variety of pancreatic enzymes such as amylase, lipase and elastase, and also being able to modulate the immune response by suppressing 


$\begin{array}{lrr}\text { 3.1.1 Ulinastatin administration began before ERCP } \\ \text { Tsujino et al } & 18 & 204 \\ \text { Yao et al } & 2 & 64 \\ \text { Park et al } & 16 & 53 \\ \text { Chen et al (Zhejiang JITCWM) } & 14 & 60 \\ \text { Chen et al (Chinese Rural Medicine) } & 16 & 60 \\ \text { Subtotal (95\% Cl) } & & 441 \\ \text { Total events } & 66 & \end{array}$

Total events

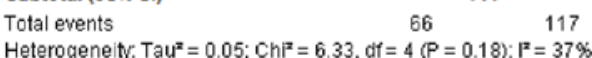

\section{$\begin{array}{llll}204 & 29 & 202 & 15.8 \%\end{array}$}

64
53

$21-60 \quad 15.5 \%$

$\begin{array}{llll}60 & 25 & 59 & 16.5 \%\end{array}$

\begin{tabular}{l}
$41-436 \quad 70.3 \%$ \\
\hline
\end{tabular}

Test for overall effect: $Z=3.05(P=0.002)$

3.1.2 Ulinastatin administration begain during ERCP

$\begin{array}{llllllll}\text { Ohwada et al } & 6 & 22 & 20 & 46 & 12.5 \% & 0.63[0.29,1.34] & 1997 \\ \text { Subtotal }(95 \% \mathrm{Cl}) & & 22 & & 46 & 12.5 \% & 0.63[0.29,1.34] & \\ \text { Total events } & 6 & & 20 & & & \end{array}$

Total events 20

Heterogeneity: Not applicable

Test for overall effect: $Z=1.21(P=0.23)$

3.1.3 Ulinastatin administration begain after ERCP

$\begin{array}{llllllll}\text { Yoo et al } & 36 & 119 & 21 & 108 & 17.2 \% & 1.56[0.97,2.49] & 2008 \\ \text { Subtotal }(95 \% \mathrm{Cl}) & & 119 & & 108 & 17.2 \% & 1.56[0.97,2.49] & \\ \text { Total events } & 36 & & 21 & & & \end{array}$

Heterogeneity: Not applicable

Test for overall effect: $Z=1.84(P=0.07)$

$\begin{array}{llrrrr}\text { Total }(95 \% \mathrm{Cl}) & 582 & 590 & \mathbf{1 0 0 . 0 \%} & \mathbf{0 . 6 7} & {[0.45,1.00]}\end{array}$

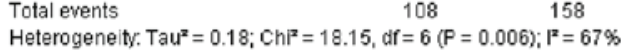

Test for overall effect: $Z=1.94(P=0.05)$

Test for subaroup differences: $\mathrm{Ch}^{2}=11.23, \mathrm{df}=2(\mathrm{P}=0.004), \mathrm{I}^{2}=82.2 \%$

$0.61[0.35,1.07] 2005$ $0.11[0.03,0.44] 2005$ $0.67[0.40,1.10] 2014$ $0.67[0.38,1.18] 2015$ $0.63[0.38,1.05] 2015$ $0.59[0.42,0.83]$

Risk Ratio Events Total Events Total Weight M.H. Random, 95\% Cl Year

\begin{tabular}{ll} 
Study or Subgroup & Events Total Events Total Weight $\mathrm{M}-\mathrm{H}$, Random, $95 \% \mathrm{Cl}$ Year \\
\hline 3.2 .1 Ulinastatin administered only before ERCP &
\end{tabular}

$\begin{array}{lccccccc}\text { 3.2.1 Ulinastatin administered only before ERCP } & & & & & \\ \text { Tsujino et al } & 18 & 204 & 29 & 202 & 22.7 \% & 0.61[0.35,1.07] & 2005 \\ \text { Subtotal }(95 \% \mathrm{Cl}) & & 204 & & 202 & 22.7 \% & 0.61[0.35,1.07] & \\ \text { Total events } & 18 & & 29 & & & \end{array}$

Heterogeneity. Not applicable 29

Test for overall effect: $Z=1.72(P=0.09)$

3.2.2 Ulinastatin administered both before and after ERCP

Yao et al

Park et al

Chen et al (Chinese Rural Medicine)

Chen et al (Zhejiang JITCWMM)

Subtotal $(95 \% \mathrm{Cl})$

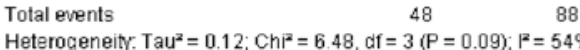

$\begin{array}{rlllr}2 & 64 & 18 & 62 & 5.3 \% \\ 16 & 53 & 24 & 53 & 253 \%\end{array}$

$\begin{array}{lllll}16 & 60 & 25 & 59 & 24.8 \%\end{array}$

$\begin{array}{lllll}14 & 60 & 21 & 60 & 21.8 \%\end{array}$

Test for overall effect: $Z=2.45(P=0.01)$

Total $(95 \% \mathrm{Cl})$

Total events

117
$0.67[0.40,1.10] 2014$ $0.63[0.38,1.05] 2015$ $0.67[0.38,1.18] 2015$ $0.56[0.35,0.89]$

Heterogeneity. $\mathrm{Tau}^{2}=0.05 ; \mathrm{Chi}^{2}=6.33, \mathrm{df}=4(\mathrm{P}=0.18) ; \mathrm{F}^{2}=37^{\circ} \%$

Test for overall effect $Z=305(P=0002)$

Test for subqroup differences: $\mathrm{Ch}^{\mathrm{z}}=0.07, \mathrm{df}=1(\mathrm{P}=0.79), \mathrm{F}^{\mathrm{a}}=0 \%$ M-H, Random, $95 \%$

C

Study or Subgroup

Ulinastatin Control Risk Ratio

Events Total Events Total Weight M-H, Random, $95 \% \mathrm{Cl}$ Year

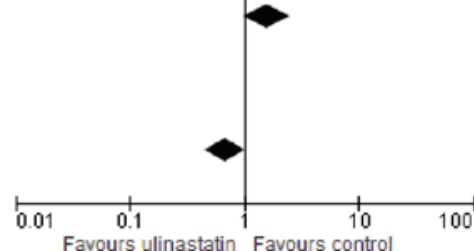

\begin{tabular}{|c|c|c|c|c|c|c|}
\hline \multicolumn{7}{|l|}{ 3.3.1 High-risk patients } \\
\hline Yoo et al & 36 & 119 & 21 & 108 & $15.4 \%$ & $1.56[0.97,2.49]$ \\
\hline $\begin{array}{l}\text { Park et al - High-risk patients } \\
\text { Subtotal }(95 \% \mathrm{Cl})\end{array}$ & 14 & $\begin{array}{r}42 \\
161\end{array}$ & 15 & $\begin{array}{r}16 \\
124\end{array}$ & $\begin{array}{l}15.7 \% \\
31.2 \%\end{array}$ & $\begin{array}{l}0.36[0.23,0.56] \\
0.74[0.16,3.53]\end{array}$ \\
\hline Total events & 50 & & 36 & & & \\
\hline \multicolumn{7}{|c|}{$\begin{array}{l}\text { Heterogeneity: Tauz }=1.21 ; \mathrm{Chi}^{\mathbf{z}}=23.08, \text { df }=1(P<0.00001) ; \mathrm{I}^{z}=96 \% \\
\text { Test for overall effect: } Z=0.37(P=0.71)\end{array}$} \\
\hline \multicolumn{7}{|l|}{ 3.3.2 Other patients } \\
\hline Onwada et al & 6 & 22 & 20 & 46 & $12.1 \%$ & $0.63[0.29,1.34]$ \\
\hline Tsujino et al & 18 & 204 & 29 & 202 & $14.5 \%$ & $0.61[0.35,1.07]$ \\
\hline Yao et al & 2 & 64 & 18 & 62 & $6.4 \%$ & $0.11[0.03,0.44]$ \\
\hline Park et al - Low-risk patients & 2 & 11 & 9 & 37 & $6.7 \%$ & $0.75[0.19,2.96]$ \\
\hline Chen et al (Chinese Rural Medicine) & 16 & 60 & 25 & 59 & $14.9 \%$ & $0.63[0.38,1.05]$ \\
\hline $\begin{array}{l}\text { Chen et al (Zhejiang JITCWMM) } \\
\text { Subtotal ( } 95 \% \text { CI) }\end{array}$ & 14 & $\begin{array}{r}60 \\
421\end{array}$ & 21 & $\begin{array}{r}60 \\
466\end{array}$ & $\begin{array}{l}14.2 \% \\
68.8 \%\end{array}$ & $\begin{array}{l}0.67[0.38,1.18] \\
0.59[0.42,0.81]\end{array}$ \\
\hline Total events & 58 & & 122 & & & \\
\hline \multicolumn{7}{|c|}{$\begin{array}{l}\text { Heterogeneity } \text { Tau }^{2}=0.03 ; \mathrm{Chi}^{2}=6.23, \mathrm{df}=5(\mathrm{P}=0.28) ; \mathrm{I}^{2}=20 \% \\
\text { Test for overall effect: } Z=3.22(P=0.001)\end{array}$} \\
\hline Total $(95 \% \mathrm{Cl})$ & & 582 & & 590 & $100.0 \%$ & $0.60[0.39,0.94]$ \\
\hline Total events & 108 & & 158 & & & \\
\hline \multicolumn{7}{|c|}{ Heterogeneity: $\mathrm{Tau}^{2}=0.27 ; \mathrm{Chi}^{2}=26.60, \mathrm{df}=7(\mathrm{P}=0.0004) ; \mathrm{I}^{2}=74 \%$} \\
\hline \multicolumn{7}{|c|}{ Test for overall effect: $Z=2.26(P=0.02)$} \\
\hline Test for subqroup differences: $\mathrm{Chi}^{2}=$ & & -0 & & & & \\
\hline
\end{tabular}

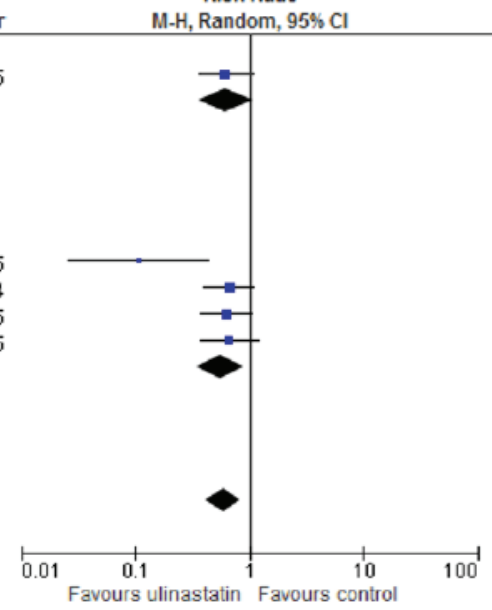

Figure 4. Incidence of post-ERCP abdominal pain in patients who received prophylactic ulinastatin vs control. (A) Analysis with stratification by whether the ulinastatin administration began prior to, during or after ERCP. (B) Analysis for patients who began ulinastatin administration prior to ERCP, further stratified by whether ulinastatin was administered only prior to ERCP or also after ERCP. (C) Analysis with patients stratified into those with high risk for post-ERCP abdominal pain and others. 


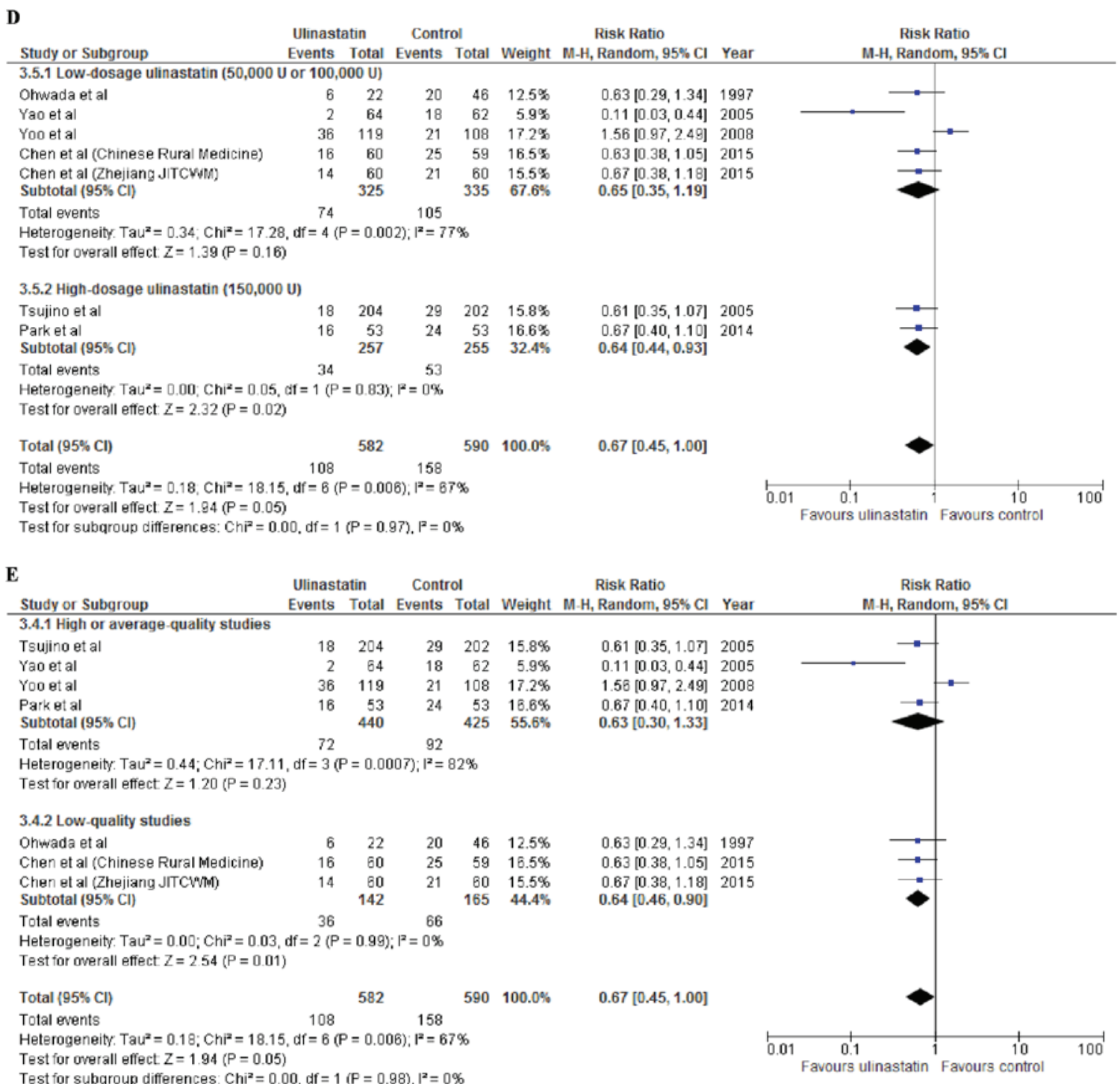

Figure 4. Continued. (D) Analysis with stratification by the dosage of ulinastatin administered: Low-dosage group (100,000 U) and high-dosage group $(150,000$ or $200,000 \mathrm{U})$. (E) Analysis stratified by the qualities of the included studies: High or average-quality studies (Jadad score, $\geq 2)$ and low-quality studies (Jadad score, <2). The squares and horizontal lines represent risk ratio and 95\% CI for included studies, respectively. CI, confidence interval; M-H, Mantel-Haenszel; ERCP, endoscopic retrograde cholangiopancreatography; df, degrees of freedom.

pro-inflammatory mediators such as interleukin- 6 and tumor necrosis factor- $\alpha$, which are associated with the severity of pancreatitis $(3,6,23)$. The present analysis supported the efficacy of ulinastatin in preventing PEP, although several conditions are required for this. First, the present analysis revealed that ulinastatin was only effective when its administration began prior to or during ERCP. Considering the fact that in pancreatitis, the acute-phase response to pancreatic injury starts immediately after pancreatic damage, the present result makes sense, since any attempt to prevent PEP must start sufficiently early so that any trypsin activation-induced downstream events are discontinued. Furthermore, the present analysis revealed that only high-dosage ulinastatin $(150,000$ or $200,000 \mathrm{U}$ ) was effective. Indeed, for a medication to work properly, a sufficient concentration in its target organ/tissue should be achieved, and in the scenario of the present study, a dosage of at least $150,000 \mathrm{U}$ is needed. In addition, the present analysis revealed that prophylactic ulinastatin was only effective in patients with a low risk for PEP; however, only 2 studies including 161 patients receiving prophylactic ulinastatin and 124 patients taking a placebo were included for analysis of high-risk patients $(8,9)$, and in one of the studies with a $72 \%$-weight in the analysis, ulinastatin was administered after ERCP at a low dose (100,000 U) (8). As revealed by the present analysis, ulinastatin administered after ERCP or at a low dose was not effective; therefore, the the question as to whether prophylactic ulinastatin may be effective in high-risk patients if administered timely and at a high dosage was not properly answered. The other study, by Park et al (9), found that ulinastatin significantly reduced the risk of PEP in high-risk patients; however, it was a small-scale study including only 42 high-risk patients receiving ulinastatin and 16 high-risk patients receiving placebo, and as such, its results are less than solid. The question as to whether ulinastatin is effective in these high-risk patients is important because after all, the overall frequency of PEP is relatively low (1-10\%) and most PEP cases are mild $(1,2)$; therefore, routine prophylactic ulinastatin administration for every patient undergoing ERCP 
does not appear to be cost-effective. However, if ulinastatin is effective in patients with a high risk for PEP, administration of ulinastatin only to high-risk patients may be more cost-effective. Although certain risk factors for PEP, including difficulty in cannulation, pre-cut sphincterotomy and pancreatic acinarization, were only identified during or after ERCP (8), other risk factors such as young age, female gender, suspected sphincter of Oddi dysfunction, previous PEP and therapeutic ERCP (9) were readily identifiable prior to ERCP. If prophylactic ulinastatin is effective in high-risk patients, pre-ERCP ulinastatin administration may reduce the risk of PEP for patients in which risk factors are identifiable prior to ERCP. The present analysis on timing of ulinastatin administration also revealed that starting ulinastatin administration during ERCP may significantly reduce the incidence of PEP; however, the present analysis only included 2 studies with 253 patients receiving ulinastatin and 205 patients receiving placebo $(7,14)$; therefore, the validity of this result was less than definite and more studies on this topic are required. If starting ulinastatin administration during ERCP is not too late to prevent PEP, patients presenting with risk factors for PEP during ERCP, such as difficult cannulation and pre-cut sphincterotomy, may be administered high-dosage ulinastatin during ERCP to prevent PEP. In summary, it is worthwhile to perform additional studies on whether prophylactic ulinastatin is effective in preventing PEP in high-risk patients and also on whether starting ulinastatin administration during ERCP may still prevent PEP in order to improve the cost-effectiveness of prophylactic ulinastatin by administering ulinastatin only to high-risk patients.

The present analysis further revealed that post-ERCP ulinastatin administration in addition to pre-ERCP ulinastatin did not have any added benefit, and that pre-ERCP high-dosage ulinastatin was effective in preventing ERCP, so repeated ERCP administration is not necessary. However, since only 2 studies with 224 patients taking ulinastatin and 222 patients taking placebo were included $(6,11)$, more studies on this topic are required to further confirm this result.

The present study also analyzed the preventive effect of ulinastatin on PEHA and post-ERCP abdominal pain, since they are possible symptoms of PEP. Asymptomatic PEHA occurs in $35-75 \%$ of patients undergoing ERCP, the degree of amylase elevation does not appear to correlate with the severity of PEP, and it is rather the timing of hyperamylasemia that has an important role in the early diagnosis of PEP $(3,8)$. Normally, PEP develops between 3-7 h post-ERCP, and an increase in serum pancreatic enzymes such as amylase and lipase may be observed 2-4 $\mathrm{h}$ after ERCP and abdominal pain starts between 3-7 h post-ERCP $(3,8,22)$. Therefore, studies on the effect of ulinastatin on preventing PEHA and abdominal pain should focus on reducing their incidence within a couple of $h$ post-ERCP.

The present analysis was limited by several factors. First and foremost, inter-study heterogeneity was present, as reflected by the impact of using a fixed-effects model on certain results. These heterogeneities may be due to multiple factors. Definitions of PEP and PEHA as well as the time-point(s) for recording abdominal pain differed among the studies. Only unification of the definitions for PEP and PEHA and also the time-point(s) of recording abdominal pain will substantially decrease the heterogeneities. The 1991 Consensus Guidelines defined PEP as a newly developed or increased abdominal pain within $24 \mathrm{~h}$ after ERCP requiring analgesic agents, accompanied by the elevation of serum amylase and/or lipase levels at least 3 times the normal upper limit $24 \mathrm{~h}$ after ERCP (24), and we recommend this definition of PEP. Another reason for such substantial heterogeneities may be the differences in study design. The present study made no adjustment for confounding effects of pancreatic stenting; recent improvements in endoscopic techniques contributed to a decreased risk of PEP in recent years, and as such, the beneficial effects associated with prophylactic ulinastatin in the later studies may be somewhat obscured.

The present study was further limited by the presence of low-quality studies, as reflected by the impact of excluding low-quality studies from the results on the effect of prophylactic ulinastatin in prevent PEHA and post-ERCP abdominal pain. However, sub-group analysis based on the quality of included studies was performed to determine the robustness and steadiness of the present results. Furthermore, possible publication bias was identified among the studies, as reflected by asymmetrical funnel plots.

At the same time, the present analysis had several strengths. First, all relevant studies not only published in English, but also in Chinese, were included, and considering the fact that a substantial portion of the studies on the effect of prophylactic ulinastatin on preventing PEP were performed in China, inclusion of studies published in Chinese improved the statistical power of the analysis. In addition, the quality of each study included was rigorously assessed and a sub-group analysis was performed based on the quality of the studies to test the validity and robustness of the present analyses. Furthermore, multiple outcome measures were used, and in addition to PEP and PEHA, the effect of ulinastatin on post-ERCP abdominal pain was also analyzed. Finally, multiple sub-group analyses were performed in order to further delineate a precise method of ulinastatin administration.

In conclusion, prophylactic ulinastatin administration significantly reduced the risk of PEP in patients with low or average risk for PEP when administered at a high dosage prior to or during ERCP. High-quality studies on high-risk patients and for ulinastatin administered during ERCP are warranted.

\section{Acknowledgements}

This study was funded by the Shanghai Medical Key Specialty Fund (grant no. ZK2015B10).

\section{References}

1. Yuhara H, Ogawa M, Kawaguchi Y, Igarashi M, Shimosegawa T and Mine T: Pharmacologic prophylaxis of post-endoscopic retrograde cholangiopancreatography pancreatitis: Protease inhibitors and NSAIDs in a meta-analysis. J Gastroenterol 49: 388-399, 2014

2. $\mathrm{Yu} \mathrm{G}, \mathrm{Li} \mathrm{S}$, Wan R, Wang $\mathrm{X}$ and $\mathrm{Hu}$ G: Nafamostat mesilate for prevention of post-ERCP pancreatitis: A meta-analysis of prospective, randomized, controlled trials. Pancreas 44: 561-569, 2015.

3. Chen S, Shi H, Zou X and Luo H: Role of ulinastatin in preventing post-endoscopic retrograde cholangiopancreatography pancreatitis: The emperor's new clothes or aladdin's magic lamp? Pancreas 39: 1231-1237, 2010. 
4. Yoo YW, Cha SW, Kim A, Na SY, Lee YW, Kim SH, Lee HIe, Lee YJ, Yang HW and Jung SH: The use of gabexate mesylate and ulinastatin for the prevention of post-endoscopic retrograde cholangiopancreatography pancreatitis. Gut Liver 6: 256-261, 2012.

5. Dumonceau JM, Andriulli A, Deviere J, Mariani A, Rigaux J, Baron TH and Testoni PA; European Society of Gastrointestina Endoscopy: European Society of Gastrointestinal Endoscopy (ESGE) guideline: Prophylaxis of post-ERCP pancreatitis. Endoscopy 42: 503-515, 2010.

6. Tsujino T, Komatsu Y, Isayama H, Hirano K, Sasahira N, Yamamoto N, Toda N, Ito Y, Nakai Y, Tada M, et al: Ulinastatin for pancreatitis after endoscopic retrograde cholangiopancreatography: A randomized, controlled trial. Clin Gastroenterol Hepatol 3: 376-383, 2005.

7. Wang J, Su J, Lu Y, Zhou H and Gong B: A randomized control study to investigate the application of ulinastatin-containing contrast medium to prevent post-ERCP pancreatitis. Hepatogastroenterology 61: 2391-2394, 2014.

8. Yoo JW, Ryu JK, Lee SH, Woo SM, Park JK, Yoon WJ, Lee JK, Lee KH, Hwang JH, Kim YT and Yoon YB: Preventive effects of ulinastatin on post-endoscopic retrograde cholangiopancreatography pancreatitis in high-risk patients: A prospective, randomized, placebo-controlled trial. Pancreas 37: 366-370, 2008

9. Park JY, Jeon TJ, Hwang MW, Sinn DH, Oh TH, Shin WC and Choi WC: Comparison between ulinastatin and nafamostat for prevention of post-endoscopic retrograde cholangiopancreatography complications: A prospective, randomized trial. Pancreatology 14: 263-267, 2014

10. Ohwada M, Watanabe N, Maeda M, Gotoh M, Teramoto J, Moriya H, Nakajima T, Okamoto T, Tsuji N, Kobayashi D and Niitsu Y: New endoscopic treatment for chronic pancreatitis, using contrast media containing ulinastatin and prednisolone. J Gastroenterol 32: 216-221, 1997.

11. Song AL, Yin LN and Kou ZM: Preventive effects of ulinastatin on post ERCP hyperamylasemia and acute pancreatitis. Lanzhou Da Xue Xue Bao (Yi Xue Ban) 31: 24-25, 2005 (In Chinese).

12. Gong P, Wang ZY,Zuo P, Gong AX,Zhao RY and Li KJ: Prevention and treatment for post ERCP pancreatitis. J Hepatobiliary Surg 12: 101-103, 2004 (In Chinese)

13. Xiong W, Yang Y, Dong K and Gong J: Clinical investigation of ulinastatin in preventing and treating hyperamylasemia and acute pancreatitis following endoscopic retrograde cholangio pancreatography. Yao Wu Yu Lin Chuang 10: 40-42, 2013 (In Chinese).
14. Wang JP, Su JG, Lu YN, Zhou HH and Gong B: Clinical randomizing control trial for the imaging of pancreatic duct and post ERCP pancreatitis: A method using a contrast medium with ulinastatin. Chin J Practical Intern Med 30: 256-257,260, 2010 (In Chinese)

15. Yao Y, Liao A, Shi W and Tan JX: Clinical observation of preventive effects of ulinastatin on post-ERCP pancreatitis. J Nanhua Univ (Med Edition) 33: 98-99, 2005 (In Chinese).

16. Chen X, Xiao C, Yan H and Li D: Prevention of post-ERCP pancreatitis: A randomized, placebo-controlled study. Zhejiang J Integr Tradit Chin Western Med 25: 686-688, 2015 (In Chinese).

17. Chen X, Xia C, Yan H, Wang GP and Li D: Comparative efficacies of ulinastatin and octreotide in preventing and treating post-ERCP pancreatitis. Zhong Guo Xiang Cun Yi Yao 22: 20-21, 2015 (In Chinese).

18. Jadad AR, Moore RA, Carroll D, Jenkinson C, Reynolds DJ, Gavaghan DJ and McQuay HJ: Assessing the quality of reports of randomized clinical trials: Is blinding necessary? Control Clin Trials 17: 1-12, 1996

19. DerSimonian R and Laird N: Meta-analysis in clinical trials. Control Clin Trials 7: 177-188, 1986.

20. Higgins J, Thompson S, Deeks J and Altman D: Statistical heterogeneity in systematic reviews of clincial trials: A critical appraisal of guidelines and practice. J Health Serv Res Policy 7: 51-61, 2002.

21. Navarro-Mateu F, Escámez T, Koenen KC, Alonso J and Sánchez-Meca J: Meta-analyses of the 5-HTTLPR polymorphism and post-traumatic stress disorder. PLoS One 8: e66227, 2013.

22. Ueki T, Otani K, Kawamoto K, Shimizu A, Shimizu A, Fujimura N, Sakaguchi S and Matsui T: Comparison between ulinastatin and gabexate mesylate for the prevention of post-endoscopic retrograde cholangiopancreatography pancreatitis: A prospective, randomized trial. J Gastroenterol 42: 161-167, 2007.

23. Uemura K, Murakami Y, Hayashidani Y, Sudo T, Hashimoto Y, Ohge $\mathrm{H}$ and Sueda T: Randomized clinical trial to assess the efficacy of ulinastatin for postoperative pancreatitis following pancreaticoduodenectomy. J Surg Oncol 98: 309-313, 2008.

24. Cotton PB, Lehman G, Vennes J, Geenen JE, Russell RC, Meyers WC, Liguory C and Nickl N: Endoscopic sphincterotomy complications and their management: An attempt at consensus. Gastrointest Endosc 37: 383-393, 1991. 